\title{
SN 2008am: A SUPER-LUMINOUS TYPE IIn SUPERNOVA
}

\author{
E. Chatzopoulos ${ }^{1}$, J. Craig Wheeler ${ }^{1}$, J. Vinko ${ }^{2}$, R. Quimby ${ }^{3}$, E. L. Robinson ${ }^{1}$, A. A. Miller ${ }^{4}$, R. J. Foley ${ }^{5,7}$,

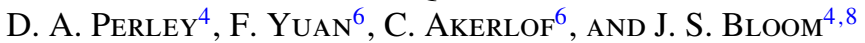 \\ ${ }^{1}$ Department of Astronomy, University of Texas at Austin, Austin, TX, USA; manolis@astro.as.utexas.edu \\ ${ }^{2}$ Department of Optics and Quantum Electronics, University of Szeged, Szeged, Hungary \\ ${ }^{3}$ Division of Physics, Mathematics and Astronomy, California Institute of Technology, Pasadena, CA 91125, USA \\ ${ }^{4}$ Department of Astronomy, University of California, Berkeley, CA 94720-3411, USA \\ ${ }^{5}$ Harvard-Smithsonian Center for Astrophysics, 60 Garden Street, Cambridge, MA 02138, USA \\ ${ }^{6}$ University of Michigan, Randall Laboratory of Physics, 450 Church Street, Ann Arbor, MI 48109-1040, USA \\ Received 2010 March 3; accepted 2011 January 18; published 2011 February 22
}

\begin{abstract}
We present observations and interpretation of the Type IIn supernova SN 2008am discovered by the ROTSE Supernova Verification Project (RSVP). SN 2008am peaked at approximately -22.3 mag at a redshift of $z=$ 0.2338 , giving it a peak luminosity of $\sim 3 \times 10^{44} \mathrm{erg} \mathrm{s}^{-1}$ and making it one of the most luminous supernovae ever observed. The total radiated energy is $\simeq 2 \times 10^{51} \mathrm{erg}$. The host galaxy appears to be an SB1 of normal luminosity $\left(M_{r^{\prime}} \sim-20\right)$ with metallicity $Z \sim 0.4 Z_{\odot}$. ROTSE upper limits and detections constrain the rise time to be $\sim 34$ days in the rest frame, significantly shorter than similar events, SN 2006gy and SN 2006tf. Photometric observations in the ultraviolet, optical, and infrared bands $\left(J, H, K_{s}\right)$ constrain the spectral energy distribution evolution. We obtained six optical spectra of the supernova, five on the early decline from maximum light and a sixth nearly a year later plus a very late time spectrum $(\sim 2 \mathrm{yr})$ of the host galaxy. The spectra show no evidence for broad supernova photospheric features in either absorption or emission at any phase. The spectra of SN 2008am show strong Balmer-line and He I $\lambda 5876$ emission with intermediate widths $(\sim 25 \AA)$ in the first $\sim 40$ days after optical maximum. The width formally corresponds to a velocity of $\sim 1000 \mathrm{~km} \mathrm{~s}^{-1}$. We examine a variety of models for the line wings and conclude that multiple scattering is most likely, implying that our spectra contain no specific information on the bulk flow velocity. We examine a variety of models for the ROTSE light curve subject to the rise time and the nature of the spectra, including radioactive decay, shocks in optically thick and optically thin circumstellar media (CSMs) and a magnetar. The most successful model is one for which the CSM is optically thick and in which diffusion of forward shock-deposited luminosity gives rise to the observed light curve. The model suggests strong mass loss and a greater contribution from the interaction of the forward shock with optically thick CSM than from the reverse shock. Diffusion of the shock-deposited energy from the forward shock is found to be important in accounting for the rising part of the light curve. Although there are differences in detail, SN 2008am appears to be closely related to other super-luminous Type IIn supernovae, SN 2006gy, SN 2006tf, and perhaps SN 2008iy, that may represent the deaths of very massive luminous-blue-variable-type progenitors and for which the luminosity is powered by the interaction of the ejecta with a dense CSM.
\end{abstract}

Key words: circumstellar matter - hydrodynamics - stars: evolution - supernovae: general - supernovae: individual (SN 2008am)

Online-only material: color figures

\section{INTRODUCTION}

The Texas Supernova Search (TSS; Quimby et al. 2005) and its successor, the ROTSE Supernova Verification Project (RSVP; Yuan et al. 2007), discovered a new class of superluminous supernovae (SLSNe). The advantage of the TSS/ RSVP project is that it is essentially free of selection bias and the limits of a targeted search. The automated wide field (3.4 deg $\left.{ }^{2}\right)$ ROTSE-III telescopes (Akerlof et al. 2003) scan the whole sky, looking for transients down to $\sim 19$ mag. They do not focus on pre-selected galaxies nor omit galaxy centers. The first TSS/ RSVP discoveries in this new class of SLSNe were SN 2005ap (Quimby et al. 2007a), SN 2006gy (Quimby 2006; Smith et al. 2007), SN 2006tf (Quimby et al. 2007b, 2007c; Smith et al. 2008), and SN 2008es (Yuan et al. 2008b; Gezari et al. 2009; Miller et al. 2009). These exceptionally luminous supernovae are rare, with an estimated rate of $\sim 2.6 \times 10^{-7}$ events $\mathrm{Mpc}^{-3} \mathrm{yr}^{-1}$

\footnotetext{
7 Clay Fellow.

8 Sloan Research Fellow.
}

(Quimby et al. 2009). The SLSNe introduced new modes of stellar death. Traditional ideas about the mechanisms that can power supernova luminosity were found to be inadequate to explain the observed properties of these events.

The small, but growing, sample of SLSNe is heterogeneous. Some show strong emission lines of hydrogen in their spectra close to maximum light (SN 2006gy, SN 2006tf, SN 2008fz, SN 2008iy) and typically belong to the Type IIn subclass; some show hydrogen in later phases and a linear decline of the light curve expressed in magnitudes (SN 2008es). Others may show no hydrogen at all (SN 2005ap, SCP06F6). For the superluminous Type IIn events, the energy generation mechanism is very likely the interaction between the ejecta and a circumstellar medium (CSM) that was shed by the progenitor star in the years prior to the explosion (Chevalier \& Fransson 2003). SN 2006gy triggered discussions about the possibility of nearby pair-instability supernovae (Smith et al. 2007). Such models proved unsatisfactory for SN 2006gy and many other SLSNe but may account for SN 2007bi (Gal-Yam et al. 2009; Young 

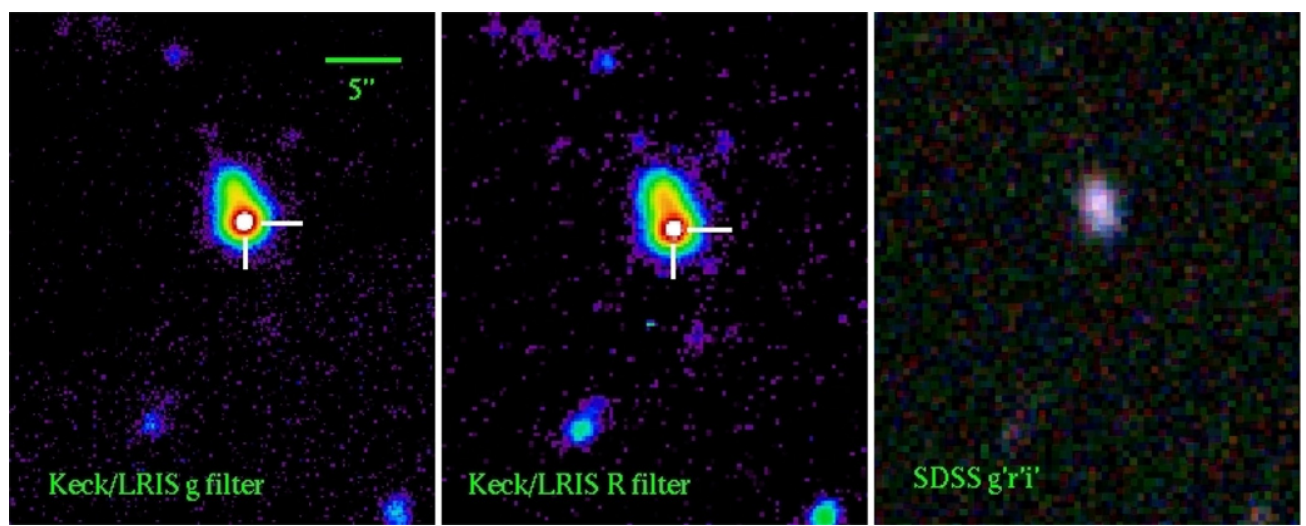

Figure 1. False-color images showing the position of SN 2008am relative to its host galaxy in the Keck/LRIS $g$ (left panel) and $R$ (middle panel) filters. Both Keck images were taken on 2008 February 12. The right panel contains the true-color SDSS image of the host (blue $=g^{\prime}$, green $=r^{\prime}$, red $=i^{\prime}$ filters). The scale is indicated in the left panel. North is up and east is to the left on all images.

(A color version of this figure is available in the online journal.)

et al. 2010). Even for some events that do not show clear signs of CSM interaction, simple radioactive decay diffusion models (Arnett 1982; Valenti et al. 2008) have proven inconsistent with the observations (Quimby et al. 2007a, 2009; Gezari et al. 2009). Other mechanisms that can account for the large luminosity have been proposed: interaction between expelled shells (Woosley et al. 2007); interaction between a gamma-ray burst (GRB)like jet and the progenitor envelope (Young et al. 2005; Gezari et al. 2009); a buried magnetar (Kasen \& Bildsten 2010; Woosley 2010); or a very energetic core-collapse explosion (Umeda \& Nomoto 2008; Moriya et al. 2010). In addition, the possibility that many Type IIn SNe (of normal or high luminosity) have been spectroscopically confused with radioquiet low-luminosity blazars has been discussed (Filippenko 1989). All recent SLSNe candidates have shown spectroscopic features that are more consistent with $\mathrm{SNe}$.

In the present work, we report on SN 2008am discovered by RSVP (Yuan et al. 2008a). The paper is organized as follows. In Section 2 we present the photometric and spectroscopic observations of SN 2008am and discuss the evolution of its spectral energy distribution (SED). In Section 3 we consider the nature of the emission-line features and models to account for the line profiles, and in Section 4 we discuss the applicability of various models to account for the light curve. Finally, in Section 5 we summarize our conclusions.

\section{OBSERVATIONS}

\subsection{The Host of SN $2008 \mathrm{am}$}

The host of SN 2008am is SDSS J122836.32+153449.6 that appears to be a faint, extended object in the Sloan Digital Sky Survey (SDSS), with an $r^{\prime}$ magnitude of $\sim 20$ (see Figure 1). The position of the centroid of the host is $\alpha=12^{\mathrm{h}} 28^{\mathrm{m}} 36^{\mathrm{s}} 3$ and $\delta=$ $+15^{\mathrm{d}} 34^{\mathrm{m}} 50^{\mathrm{s}}$ and its redshift is $z=0.2338$ (Yuan et al. 2008a; Section 2.4). We estimate the luminosity distance of the SN to be $1130 \mathrm{Mpc}$ assuming a $\Lambda \mathrm{CDM}$ cosmology with $\Omega_{\Lambda}=0.73$, $\Omega_{M}=0.27$, and $H_{0}=73 \mathrm{~km} \mathrm{~s}^{-1} \mathrm{Mpc}^{-1}$. On that basis, the host has an absolute magnitude $M_{r^{\prime}} \sim-20$ mag and, thus, is not a dwarf galaxy. The morphology of this galaxy is unknown, but the shape of its SDSS photometric SED and the optical spectrum showing narrow emission features of $\mathrm{H},[\mathrm{N} \mathrm{II}]$, [O II] and [O III] agree well with an SB1 galaxy template spectrum (Figure 9; Section 2.4). The metallicity of the host, estimated from the flux ratios of these narrow emission features, is sub-solar

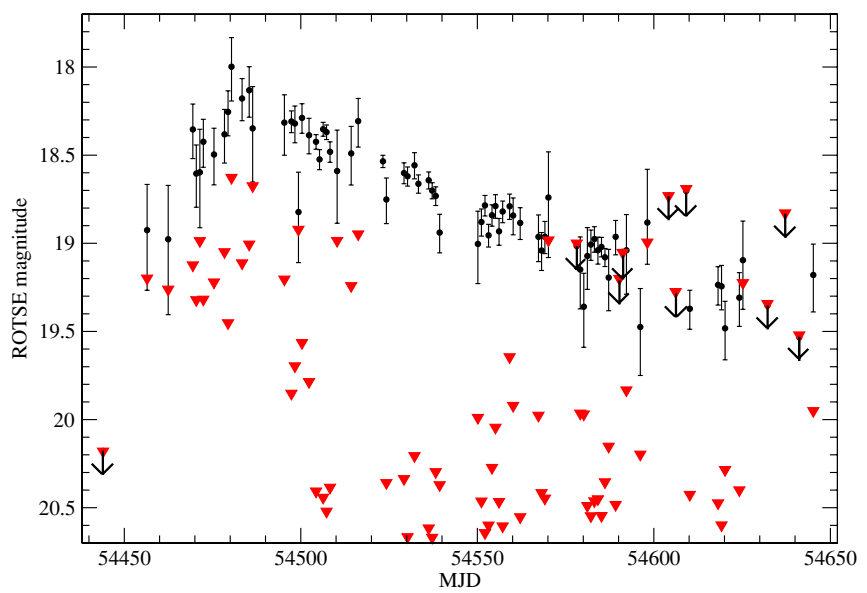

Figure 2. ROTSE unfiltered light curve of SN 2008am. The red-filled triangles represent the sensitivity limits. Upper limits lie on their contemporaneous sensitivity points and are denoted by downward arrows.

(A color version of this figure is available in the online journal.)

$\left(Z \sim 0.4 Z_{\odot}\right.$ ), similar to the hosts of other SLSNe (Neill et al. 2011; Stoll et al. 2010; Section 2.4).

\subsection{Imaging and Photometry}

SN 2008am was discovered in unfiltered ROTSE-IIIb images on 2008 January 10.4 UT (MJD = 54475.4; Yuan et al. 2008a) when it had an unfiltered magnitude of 18.7. The position of the SN in the ROTSE images was determined to be $\alpha=$ $12^{\mathrm{h}} 28^{\mathrm{m}} 36^{\mathrm{s}} .25$ and $\delta=+15^{\mathrm{d}} 34^{\mathrm{m}} 49^{\mathrm{s}}$. 1 , slightly offset from the center of its host that appears in the SDSS catalog (Figure 1). ROTSE-IIIb continued to gather unfiltered photometric data for $\sim 200$ days after the discovery. The ROTSE-IIIb photometry is summarized in Table 1. The ROTSE data are shown in Figure 2 along with the detection limits over the course of the photometry. The ROTSE-IIIb photometry includes an early upper limit and data points during the rising phase of the supernova light curve that can be used to constrain the explosion date of the SN. To determine the explosion date, we converted the ROTSE magnitudes to luminosities assuming zero bolometric correction, and fit radioactive decay diffusion models (Section 4.1) to the resulting light curve. The fitting parameters are the effective diffusion time, the nickel mass, the explosion date, and a parameter that controls the gamma-ray leakage. The best-fit radioactive-decay 
Table 1

ROTSE-IIIb Unfiltered Photometry of SN 2008am

\begin{tabular}{|c|c|c|c|}
\hline $\mathrm{MJD}^{\mathrm{a}}$ & UT Date & Magnitude $^{\mathrm{b}}$ & Error \\
\hline 54436.13 & 2007 Dec 2.13 & $(20.17)$ & \\
\hline 54443.13 & 2007 Dec 9.13 & 18.92 & 0.30 \\
\hline 54448.63 & 2007 Dec 14.13 & 18.97 & 0.36 \\
\hline 54469.54 & 2008 Jan 4.54 & 18.35 & 0.15 \\
\hline 54470.54 & 2008 Jan 5.54 & 18.60 & 0.17 \\
\hline 54471.54 & 2008 Jan 6.54 & 18.59 & 0.27 \\
\hline 54472.54 & 2008 Jan 7.54 & 18.42 & 0.13 \\
\hline 54475.41 & 2008 Jan 10.41 & 18.49 & 0.16 \\
\hline 54478.54 & 2008 Jan 13.54 & 18.38 & 0.15 \\
\hline 54479.54 & 2008 Jan 14.54 & 18.25 & 0.12 \\
\hline 54480.36 & $2008 \operatorname{Jan} 15.36$ & 17.99 & 0.17 \\
\hline 54483.54 & 2008 Jan 18.54 & 18.17 & 0.12 \\
\hline 54485.54 & 2008 Jan 20.54 & 18.13 & 0.14 \\
\hline 54486.54 & 2008 Jan 21.54 & 18.34 & 0.27 \\
\hline 54495.54 & 2008 Jan 30.54 & 18.31 & 0.17 \\
\hline 54497.54 & 2008 Feb 1.54 & 18.30 & 0.06 \\
\hline 54498.54 & 2008 Feb 2.54 & 18.32 & 0.10 \\
\hline 54499.95 & 2008 Feb 3.95 & 18.82 & 0.25 \\
\hline 54500.95 & 2008 Feb 4.95 & 18.28 & 0.08 \\
\hline 54502.95 & 2008 Feb 6.95 & 18.38 & 0.10 \\
\hline 54504.95 & 2008 Feb 8.95 & 18.42 & 0.04 \\
\hline 54505.95 & 2008 Feb 9.95 & 18.52 & 0.05 \\
\hline 54506.95 & 2008 Feb 10.95 & 18.35 & 0.03 \\
\hline 54507.95 & 2008 Feb 11.95 & 18.36 & 0.04 \\
\hline 54508.95 & 2008 Feb 12.95 & 18.48 & 0.05 \\
\hline 54510.95 & 2008 Feb 14.95 & 18.59 & 0.26 \\
\hline 54514.95 & 2008 Feb 18.95 & 18.49 & 0.16 \\
\hline 54516.95 & 2008 Feb 20.95 & 18.30 & 0.13 \\
\hline 54523.95 & 2008 Feb 27.95 & 18.53 & 0.03 \\
\hline 54524.95 & 2008 Feb 28.95 & 18.75 & 0.12 \\
\hline 54529.41 & 2008 Mar 4.41 & 18.60 & 0.05 \\
\hline 54530.41 & 2008 Mar 5.41 & 18.61 & 0.05 \\
\hline 54532.41 & 2008 Mar 7.41 & 18.55 & 0.01 \\
\hline 54533.41 & 2008 Mar 8.41 & 18.66 & 0.05 \\
\hline 54536.41 & 2008 Mar 11.41 & 18.64 & 0.04 \\
\hline 54537.41 & 2008 Mar 12.41 & 18.70 & 0.04 \\
\hline 54538.41 & 2008 Mar 13.41 & 18.73 & 0.05 \\
\hline 54539.41 & 2008 Mar 14.41 & 18.93 & 0.10 \\
\hline 54550.41 & 2008 Mar 25.41 & 19.00 & 0.20 \\
\hline 54551.41 & 2008 Mar 26.41 & 18.87 & 0.07 \\
\hline 54552.41 & 2008 Mar 27.41 & 18.78 & 0.05 \\
\hline 54553.41 & 2008 Mar 28.41 & 18.95 & 0.07 \\
\hline 54554.41 & 2008 Mar 29.41 & 18.84 & 0.06 \\
\hline 54555.41 & 2008 Mar 30.41 & 18.78 & 0.06 \\
\hline 54556.41 & 2008 Mar 31.41 & 18.93 & 0.07 \\
\hline 54557.41 & 2008 Apr 1.41 & 18.81 & 0.06 \\
\hline 54559.83 & 2008 Apr 3.83 & 18.79 & 0.07 \\
\hline 54560.83 & 2008 Apr 4.83 & 18.84 & 0.10 \\
\hline 54562.83 & 2008 Apr 6.83 & 18.88 & 0.09 \\
\hline 54567.83 & 2008 Apr 11.83 & 18.96 & 0.13 \\
\hline 54568.83 & 2008 Apr 12.83 & 19.04 & 0.10 \\
\hline 54569.83 & 2008 Apr 13.83 & 18.96 & 0.09 \\
\hline 54570.83 & 2008 Apr 14.83 & 18.74 & 0.29 \\
\hline 54578.83 & 2008 Apr 22.83 & (19.01) & $\ldots$ \\
\hline 54579.83 & 2008 Apr 23.83 & 19.14 & 0.20 \\
\hline 54580.83 & 2008 Apr 24.83 & 19.35 & 0.20 \\
\hline 54581.83 & 2008 Apr 25.83 & 19.07 & 0.17 \\
\hline 54582.83 & 2008 Apr 26.83 & 19.00 & 0.08 \\
\hline 54583.83 & 2008 Apr 27.83 & 18.97 & 0.07 \\
\hline 54584.83 & 2008 Apr 28.83 & 19.03 & 0.07 \\
\hline 54585.83 & 2008 Apr 29.83 & 19.02 & 0.05 \\
\hline 54586.83 & 2008 Apr 30.83 & 19.07 & 0.05 \\
\hline 54587.83 & 2008 May 1.83 & 19.19 & 0.17 \\
\hline 54589.24 & 2008 May 3.24 & 18.96 & 0.09 \\
\hline 54590.24 & 2008 May 4.24 & $(19.20)$ & . . \\
\hline 54591.24 & 2008 May 5.24 & (19.04) & \\
\hline
\end{tabular}

Table 1

(Continued)

\begin{tabular}{llcc}
\hline \hline MJD $^{\mathrm{a}}$ & \multicolumn{1}{c}{ UT Date } & Magnitude $^{\mathrm{b}}$ & Error \\
\hline 54592.24 & 2008 May 6.24 & 19.03 & 0.22 \\
54596.24 & 2008 May 10.24 & 19.47 & 0.24 \\
54598.24 & 2008 May 12.24 & 18.88 & 0.26 \\
54604.24 & 2008 May 18.24 & $(18.72)$ & $\ldots$ \\
54606.24 & 2008 May 20.24 & $(19.27)$ & $\ldots$ \\
54609.24 & 2008 May 23.24 & $(18.70)$ & $\ldots$ \\
54610.24 & 2008 May 24.24 & 19.37 & 0.11 \\
54618.24 & 2008 Jun 1.24 & 19.23 & 0.10 \\
54619.65 & 2008 Jun 2.65 & 19.24 & 0.12 \\
54620.65 & 2008 Jun 3.65 & 19.48 & 0.16 \\
54624.65 & 2008 Jun 7.65 & 19.30 & 0.15 \\
54625.65 & 2008 Jun 8.65 & 19.09 & 0.25 \\
54632.65 & 2008 Jun 15.65 & $(19.35)$ & $\ldots$ \\
54637.65 & 2008 Jun 20.65 & $(18.82)$ & $\ldots$ \\
54641.65 & 2008 Jun 24.65 & $(19.53)$ & $\ldots$ \\
54645.65 & 2008 Jun 28.65 & 19.17 & 0.19 \\
\hline
\end{tabular}

Notes.

${ }^{a}$ MJD values refer to the observer frame.

b Observed value; not corrected for extinction. The values in parentheses represent upper limits.

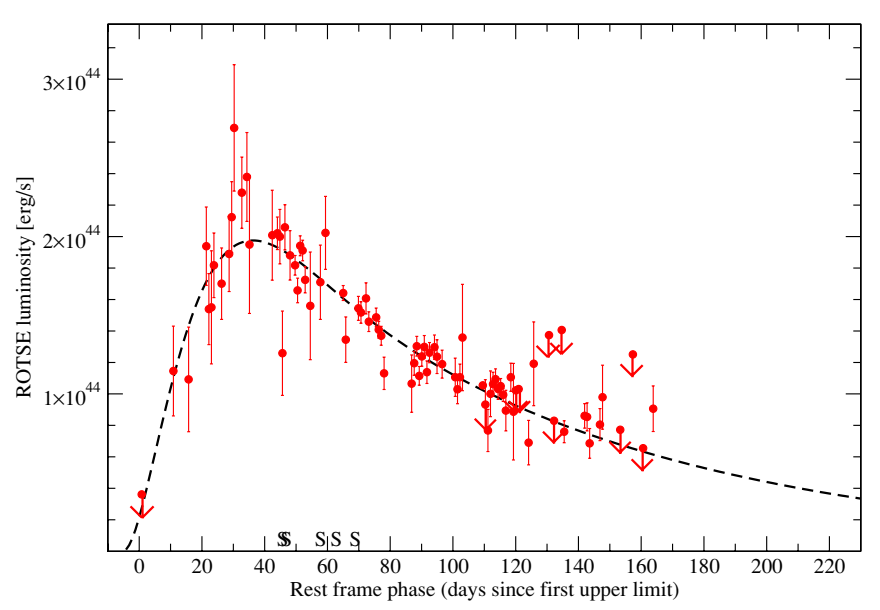

Figure 3. Fit of a simple radioactive-decay diffusion model to the ROTSE light curve of SN 2008am (filled circles). The "S" letters above the time axis denote the spectroscopic epochs. The best-fitting explosion date is MJD 54438.8, 5.2 days before the first upper limit, 14 days before the first detection, and 34 days before maximum in the rest frame. The derived nickel and ejecta mass are $M_{\mathrm{Ni}}=19 M_{\odot}$ and $M_{e j}=0.2 M_{\odot}$ (for Thompson scattering opacity $\kappa=$ $0.4 \mathrm{~cm}^{2} \mathrm{~g}^{-1}$ and velocity $v_{\mathrm{sh}}=1000 \mathrm{~km} \mathrm{~s}^{-1}$ ). The model provides a means to constrain the explosion date but is clearly not a valid physical model for the explosion (see the text).

(A color version of this figure is available in the online journal.)

diffusion model is shown in Figure 3. This fitting process yields an explosion date of $\mathrm{MJD}_{\text {expl }}=54438.8 \pm 1$, approximately 18 days prior to the first real detection in the observed frame and about 14 days in the rest frame. We note that this model was employed only to determine the explosion date of the SN and may not account for the real physical situation in SN 2008am (see Section 4.1). The ROTSE-IIIb maximum occurred at MJD = 54480.4 (2008 January 15.0), about 34 rest-frame days after the explosion.

At the distance of $1130 \mathrm{Mpc}$ for the ROTSE unfiltered peak magnitude of $18.0 \mathrm{mag}$, the absolute ROTSE peak magnitude of SN 2008am is $-22.3 \mathrm{mag}$ (uncorrected for extinction). The ROTSE response curve peaks in the red and it is calibrated to 
Table 2

PAIRITEL IR Photometry of SN 2008am

\begin{tabular}{lcccc}
\hline \hline MJD $^{\mathrm{a}}$ & UT Date & $J$ Band $^{\mathrm{b}}$ & $H$ Band $^{\mathrm{b}}$ & $K_{s}$ Band $^{\mathrm{b}}$ \\
\hline 54499.95 & 2008 Feb 3.95 & $17.65(0.12)$ & $16.96(0.22)$ & $16.78(0.31)$ \\
54502.95 & 2008 Feb 6.95 & $17.64(0.14)$ & $17.37(0.27)$ & $16.97(0.30)$ \\
54503.95 & 2008 Feb 7.95 & $17.59(0.14)$ & $17.26(0.21)$ & $16.69(0.22)$ \\
54504.95 & 2008 Feb 8.95 & $17.78(0.10)$ & $17.40(0.24)$ & $17.15(0.35)$ \\
54505.95 & 2008 Feb 9.95 & $17.60(0.09)$ & $17.16(0.17)$ & $16.61(0.17)$ \\
54506.95 & 2008 Feb 10.95 & $17.61(0.14)$ & $17.20(0.21)$ & $17.01(0.34)$ \\
54507.95 & 2008 Feb 11.95 & $17.62(0.12)$ & $17.20(0.22)$ & $16.88(0.31)$ \\
54508.95 & 2008 Feb 12.95 & $17.72(0.06)$ & $17.18(0.10)$ & $16.71(0.21)$ \\
54509.95 & 2008 Feb 13.95 & $17.62(0.18)$ & $17.20(0.18)$ & $16.78(0.30)$ \\
54510.95 & 2008 Feb 14.95 & $17.67(0.10)$ & $17.45(0.27)$ & $16.63(0.21)$ \\
54513.95 & 2008 Feb 17.95 & $17.60(0.08)$ & $17.38(0.15)$ & $17.06(0.31)$ \\
54514.95 & 2008 Feb 18.95 & $17.59(0.10)$ & $17.11(0.14)$ & $16.70(0.20)$ \\
54515.95 & 2008 Feb 19.95 & $17.73(0.11)$ & $17.26(0.16)$ & $16.89(0.20)$ \\
54516.95 & 2008 Feb 20.95 & $17.67(0.16)$ & $17.45(0.23)$ & $16.91(0.34)$ \\
54520.95 & 2008 Feb 24.95 & $17.68(0.08)$ & $17.00(0.12)$ & $16.67(0.19)$ \\
54521.95 & 2008 Feb 25.95 & $17.47(0.11)$ & $17.63(0.35)$ & $16.68(0.25)$ \\
54522.95 & 2008 Feb 26.95 & $17.75(0.09)$ & $17.30(0.16)$ & $16.96(0.27)$ \\
54523.95 & 2008 Feb 27.95 & $17.77(0.11)$ & $17.13(0.15)$ & $16.74(0.27)$ \\
54524.95 & 2008 Feb 28.95 & $17.63(0.07)$ & $17.15(0.15)$ & $17.08(0.24)$ \\
\hline
\end{tabular}

Notes.

${ }^{\text {a }}$ MJD values refer to the observer frame.

${ }^{b}$ Observed value; not corrected for extinction. The numbers in the parentheses represent the estimated errors of the measured values.

the USNO-B1.0 system (Smith et al. 2003). There is always a slight offset from a true $R$ magnitude due to the fact that the shape of a supernova SED is very different from the reference stars used by ROTSE, but, to a good approximation, this absolute peak magnitude is close to the real absolute $R$ magnitude of the event. This establishes that SN 2008am is a super-luminous event; one of the most luminous supernovae ever discovered, placing the supernova in the family of SLSNe together with SN 1992ar (Clocchiatti et al. 2000), SN 1999as (Hatano et al. 2001), SN 2003ma (Rest et al. 2009), SN 2005ap, SN 2006gy, SN 2006tf, SN 2008es, SN 2008fz (Drake et al. 2010), SN 2008iy (Miller et al. 2010), SN 2007bi (Gal-Yam et al. 2009), SCP06F6 (Barbary et al. 2009), and recently discovered luminous PanSTARRS transients (SN 2009kf, Botticella et al. 2010; SN 2010gx, Pastorello et al. 2010).

SN 2008am was followed up with photometric observations ranging from the ultraviolet (UV) through the infrared (IR). The Peters Automated Infrared Imaging Telescope (PAIRITEL; Bloom et al. 2006) obtained $J$-, $H$-, and $K_{s}$-band photometry over a 25 day period (Table 2 ). The PAIRITEL $J, H$, and $K_{s}$ fluxes are calibrated to the Two Micron All Sky Survey (2MASS) catalog (Skrutskie et al. 2006). The infrared light curves of SN 2008am over this phase appear to be flat and are probably heavily contaminated by the host. In the $K_{s}$-band, especially, the detections are most probably indicative of the host rather than the SN. Thus, we refer to them only as upper limits throughout this work. Although the infrared observations were obtained for only a small portion of the life of the $\mathrm{SN}$, they can be used to better constrain the SED toward the infrared region for some phases. Contribution by dust IR radiation to the observed $J$, $H$, and $K_{s}$ fluxes cannot be ruled out, but we have made no allowance for that process.

The Palomar 60 inch (P60) telescope obtained optical photometry in the bands $g, r, i^{\prime}$, and $z^{\prime}$ for a period of $\sim 330$ days in the rest frame. Photometry on the P60 frames was performed
Table 3

Northern Standard Stars Used for the P60 Calibration

\begin{tabular}{lcccc}
\hline \hline ID & $g^{\prime}$ & $r^{\prime}$ & $i^{\prime}$ & $z^{\prime}$ \\
\hline SDSS J122838.04+153354.4 & 17.82 & 17.05 & 16.74 & 16.60 \\
SDSS J122833.68+153505.2 & 18.18 & 17.27 & 16.95 & 16.79 \\
SDSS J122825.56+153450.2 & 17.07 & 16.15 & 15.85 & 15.69 \\
SDSS J122851.30+153427.7 & 17.62 & 17.37 & 17.27 & 17.22 \\
SDSS J122849.58+153536.8 & 16.33 & 15.95 & 15.81 & 15.75 \\
\hline
\end{tabular}

Note. The magnitudes have been tied to the SDSS $g^{\prime} r^{\prime} i^{\prime} z^{\prime}$ system.

using the aperture photometry routines in IRAF. ${ }^{9}$ The aperture radius was set to be 20 pixels ( 7.57 arcsec), and the background level was measured in an annulus with 30 pixels (11.36 arcsec) inner and 50 pixels (18.93 arcsec) outer radii, centered on the source. The P60 data were calibrated via five local tertiary standard stars having Sloan $g^{\prime}, r^{\prime}, i^{\prime}$, and $z^{\prime}$ magnitudes in the SDSS catalog (Table 3). The applied P60 filters were $g$ and $r$ (similar to the Thuan-Gunn filters; Thuan \& Gunn 1976), and $i^{\prime}$ and $z^{\prime}$ that resemble the SDSS filters (Fukugita et al. 1996), although systematic differences exist between the P60 filters and their standard counterparts (Cenko et al. 2006). Due to the lack of standard fields observed simultaneously with the SN field, only approximate calibration of the P60 photometry was possible. As a first approximation, the SN magnitudes were tied to the $g^{\prime}$, $r^{\prime}, i^{\prime}$, and $z^{\prime}$ magnitudes of the local tertiary standards assuming only a zero-point shift and no color term. Table 4 details the results of the P60 photometry of SN 2008am. The error caused by the neglect of the color term was investigated by comparing the observed differential magnitudes and colors of the local standards with their cataloged magnitudes. Only the data from the $g$ filter are affected by the lack of the color term, systematically at a level of $\sim 0.1 \mathrm{mag}$. No significant magnitude shifts were detected in any other filters. The resulting $g^{\prime}, r^{\prime}, i^{\prime}$, and $z^{\prime}$ AB magnitudes of SN 2008am were then transformed to fluxes adopting the filter parameters and flux zero points of Cenko et al. (2009). The lack of the color term caused less than $1 \sigma$ error in the $\mathrm{g}^{\prime}$ fluxes, where $\sigma$ is the random error of the photometry. The SN fluxes were subsequently corrected for host contamination and interstellar reddening. The host correction was done by removing the host fluxes obtained from SDSS. For the interstellar reddening we used the interstellar absorption maps of Schlegel et al. (1998), giving $E(B-V)_{\mathrm{MW}}=0.025 \mathrm{mag}$ and the Milky Way reddening law parameterized by Fitzpatrick \& Massa (2007) adopting $R_{V}=3.1$. Throughout this work, we use only the Milky Way reddening correction to obtain the final photometry for SN 2008am. The 50-180 day slope of the P60 $g, r, i^{\prime}$ light curve is estimated to be $0.0065 \pm 0.0006$ mag day $^{-1}$. The $z^{\prime}$ light curve is somewhat flatter over the same period.

Swift photometry was obtained by the Ultraviolet/Optical Telescope (UVOT; Roming et al. 2005) covering the first $\sim 80$ rest-frame days after maximum. The conversions between Swift magnitudes and fluxes were computed based on the calibration using the Pickles stellar templates (Poole et al. 2008) instead of the GRB templates included in the Swift CALDB. The result of the Swift photometry is detailed in Table 5. In the latest three epochs, the $U V W 1, U V M 2$, and $U V W 2$ fluxes seem to level off. This may be due to the increasing contribution from the flux of the host galaxy relative to the decreasing SN flux at later epochs.

\footnotetext{
9 IRAF is distributed by the National Optical Astronomy Observatory, which is operated by the Association of Universities for Research in Astronomy (AURA) under cooperative agreement with the National Science Foundation.
} 
Table 4

P60 Photometry of SN 2008am

\begin{tabular}{|c|c|c|c|c|}
\hline $\mathrm{MJD}^{\mathrm{a}}$ & UT Date & Filter & Magnitude $^{\mathrm{b}}$ & Error \\
\hline 54496.54 & 2008 Jan 31.54 & $g^{\prime}$ & 18.78 & 0.11 \\
\hline 54505.95 & 2008 Feb 9.95 & $g^{\prime}$ & 18.86 & 0.10 \\
\hline 54508.95 & 2008 Feb 12.95 & $g^{\prime}$ & 18.89 & 0.11 \\
\hline 54509.95 & 2008 Feb 13.95 & $g^{\prime}$ & 18.89 & 0.11 \\
\hline 54513.95 & 2008 Feb 17.95 & $g^{\prime}$ & 19.05 & 0.15 \\
\hline 54522.95 & 2008 Feb 26.95 & $g^{\prime}$ & 19.03 & 0.11 \\
\hline 54523.95 & 2008 Feb 27.95 & $g^{\prime}$ & 18.96 & 0.10 \\
\hline 54524.95 & 2008 Feb 28.95 & $g^{\prime}$ & 18.93 & 0.09 \\
\hline 54525.95 & 2008 Feb 29.95 & $g^{\prime}$ & 18.87 & 0.11 \\
\hline 54531.41 & 2008 Mar 6.41 & $g^{\prime}$ & 19.17 & 0.12 \\
\hline 54533.41 & 2008 Mar 8.41 & $g^{\prime}$ & 19.09 & 0.11 \\
\hline 54535.41 & 2008 Mar 10.41 & $g^{\prime}$ & 19.15 & 0.13 \\
\hline 54537.41 & 2008 Mar 12.41 & $g^{\prime}$ & 19.54 & 0.14 \\
\hline 54538.41 & 2008 Mar 13.41 & $g^{\prime}$ & 19.21 & 0.11 \\
\hline 54538.41 & 2008 Mar 13.41 & $g^{\prime}$ & 19.40 & 0.18 \\
\hline 54539.41 & 2008 Mar 14.41 & $g^{\prime}$ & 19.40 & 0.14 \\
\hline 54543.41 & 2008 Mar 18.41 & $g^{\prime}$ & 19.58 & 0.30 \\
\hline 54550.41 & 2008 Mar 25.41 & $g^{\prime}$ & 19.45 & 0.12 \\
\hline 54550.41 & 2008 Mar 25.41 & $g^{\prime}$ & 19.27 & 0.12 \\
\hline 54551.41 & 2008 Mar 26.41 & $g^{\prime}$ & 19.19 & 0.12 \\
\hline 54554.41 & 2008 Mar 29.41 & $g^{\prime}$ & 19.43 & 0.12 \\
\hline 54556.41 & 2008 Mar 31.41 & $g^{\prime}$ & 19.19 & 0.11 \\
\hline 54557.83 & 2008 Apr 1.83 & $g^{\prime}$ & 19.46 & 0.12 \\
\hline 54558.83 & 2008 Apr 2.83 & $g^{\prime}$ & 19.23 & 0.09 \\
\hline 54560.83 & 2008 Apr 4.83 & $g^{\prime}$ & 19.32 & 0.12 \\
\hline 54561.83 & 2008 Apr 5.83 & $g^{\prime}$ & 19.45 & 0.11 \\
\hline 54561.83 & 2008 Apr 5.83 & $g^{\prime}$ & 19.36 & 0.13 \\
\hline 54562.83 & 2008 Apr 6.83 & $g^{\prime}$ & 19.31 & 0.10 \\
\hline 54563.83 & 2008 Apr 7.83 & $g^{\prime}$ & 19.38 & 0.12 \\
\hline 54564.83 & 2008 Apr 8.83 & $g^{\prime}$ & 19.27 & 0.15 \\
\hline 54566.83 & 2008 Apr 10.83 & $g^{\prime}$ & 19.45 & 0.13 \\
\hline 54568.83 & 2008 Apr 12.83 & $g^{\prime}$ & 19.54 & 0.13 \\
\hline 54570.83 & 2008 Apr 14.83 & $g^{\prime}$ & 19.33 & 0.19 \\
\hline 54582.83 & 2008 Apr 26.83 & $g^{\prime}$ & 19.28 & 0.19 \\
\hline 54584.83 & 2008 Apr 28.83 & $g^{\prime}$ & 19.33 & 0.15 \\
\hline 54588.24 & 2008 May 2.24 & $g^{\prime}$ & 19.76 & 0.13 \\
\hline 54590.24 & 2008 May 4.24 & $g^{\prime}$ & 19.17 & 0.13 \\
\hline 54593.24 & 2008 May 7.24 & $g^{\prime}$ & 19.61 & 0.12 \\
\hline 54594.24 & 2008 May 8.24 & $g^{\prime}$ & 19.50 & 0.13 \\
\hline 54596.24 & 2008 May 10.24 & $g^{\prime}$ & 19.53 & 0.14 \\
\hline 54598.24 & 2008 May 12.24 & $g^{\prime}$ & 19.40 & 0.17 \\
\hline 54615.24 & 2008 May 29.24 & $g^{\prime}$ & 19.56 & 0.16 \\
\hline 54617.24 & 2008 May 31.24 & $g^{\prime}$ & 19.81 & 0.16 \\
\hline 54618.65 & 2008 Jun 1.65 & $g^{\prime}$ & 18.90 & 0.19 \\
\hline 54619.65 & 2008 Jun 2.65 & $g^{\prime}$ & 19.47 & 0.15 \\
\hline 54624.65 & 2008 Jun 7.65 & $g^{\prime}$ & 19.62 & 0.16 \\
\hline 54625.65 & 2008 Jun 8.65 & $g^{\prime}$ & 19.46 & 0.15 \\
\hline 54626.65 & 2008 Jun 9.65 & $g^{\prime}$ & 19.57 & 0.19 \\
\hline 54628.65 & 2008 Jun 11.65 & $g^{\prime}$ & 19.61 & 0.21 \\
\hline 54638.65 & 2008 Jun 21.65 & $g^{\prime}$ & 18.94 & 0.21 \\
\hline 54640.65 & 2008 Jun 23.65 & $g^{\prime}$ & 19.52 & 0.13 \\
\hline 54642.65 & 2008 Jun 25.65 & $g^{\prime}$ & 19.53 & 0.23 \\
\hline 54645.65 & 2008 Jun 28.65 & $g^{\prime}$ & 19.50 & 0.18 \\
\hline 54648.06 & 2008 Jul 1.06 & $g^{\prime}$ & 19.56 & 0.19 \\
\hline 54799.19 & 2008 Nov 27.19 & $g^{\prime}$ & 19.68 & 0.16 \\
\hline 54805.13 & 2008 Dec 5.13 & $g^{\prime}$ & 20.03 & 0.18 \\
\hline 54811.13 & 2008 Dec 1.13 & $g^{\prime}$ & 19.81 & 0.19 \\
\hline 54836.54 & 2009 Jan 5.54 & $g^{\prime}$ & 20.23 & 0.18 \\
\hline 54836.54 & 2009 Jan 5.54 & $g^{\prime}$ & 20.26 & 0.17 \\
\hline 54496.54 & 2008 Jan 31.54 & $r^{\prime}$ & 18.58 & 0.10 \\
\hline 54505.95 & 2008 Feb 9.95 & $r^{\prime}$ & 18.60 & 0.05 \\
\hline 54508.95 & 2008 Feb 12.95 & $r^{\prime}$ & 18.60 & 0.05 \\
\hline 54509.95 & 2008 Feb 13.95 & $r^{\prime}$ & 18.50 & 0.07 \\
\hline 54513.95 & 2008 Feb 17.95 & $r^{\prime}$ & 18.68 & 0.08 \\
\hline 54515.95 & 2008 Feb 19.95 & $r^{\prime}$ & 18.39 & 0.14 \\
\hline 54522.95 & 2008 Feb 26.95 & $r^{\prime}$ & 18.66 & 0.08 \\
\hline
\end{tabular}

Table 4

(Continued)

\begin{tabular}{|c|c|c|c|c|}
\hline $\mathrm{MJD}^{\mathrm{a}}$ & UT Date & Filter & Magnitude $^{\mathrm{b}}$ & Error \\
\hline 54523.95 & 2008 Feb 27.95 & $r^{\prime}$ & 18.76 & 0.07 \\
\hline 54524.95 & 2008 Feb 28.95 & $r^{\prime}$ & 18.70 & 0.05 \\
\hline 54525.95 & 2008 Feb 29.95 & $r^{\prime}$ & 18.77 & 0.05 \\
\hline 54531.41 & 2008 Mar 6.41 & $r^{\prime}$ & 18.72 & 0.06 \\
\hline 54533.41 & 2008 Mar 8.41 & $r^{\prime}$ & 18.73 & 0.08 \\
\hline 54535.41 & 2008 Mar 10.41 & $r^{\prime}$ & 18.82 & 0.07 \\
\hline 54537.41 & 2008 Mar 12.41 & $r^{\prime}$ & 18.82 & 0.07 \\
\hline 54538.41 & 2008 Mar 13.41 & $r^{\prime}$ & 18.89 & 0.08 \\
\hline 54538.41 & 2008 Mar 13.41 & $r^{\prime}$ & 19.00 & 0.07 \\
\hline 54539.41 & 2008 Mar 14.41 & $r^{\prime}$ & 18.78 & 0.06 \\
\hline 54543.41 & 2008 Mar 18.41 & $r^{\prime}$ & 18.95 & 0.12 \\
\hline 54544.41 & 2008 Mar 19.41 & $r^{\prime}$ & 19.13 & 0.16 \\
\hline 54545.41 & 2008 Mar 20.41 & $r^{\prime}$ & 18.79 & 0.21 \\
\hline 54546.41 & 2008 Mar 21.41 & $r^{\prime}$ & 18.42 & 0.19 \\
\hline 54549.41 & 2008 Mar 24.41 & $r^{\prime}$ & 18.86 & 0.14 \\
\hline 54550.41 & 2008 Mar 25.41 & $r^{\prime}$ & 18.56 & 0.10 \\
\hline 54551.41 & 2008 Mar 26.41 & $r^{\prime}$ & 19.00 & 0.06 \\
\hline 54554.41 & 2008 Mar 29.41 & $r^{\prime}$ & 19.02 & 0.07 \\
\hline 54554.41 & 2008 Mar 29.41 & $r^{\prime}$ & 19.05 & 0.07 \\
\hline 54556.41 & 2008 Mar 31.41 & $r^{\prime}$ & 18.90 & 0.07 \\
\hline 54557.83 & 2008 Apr 1.83 & $r^{\prime}$ & 18.82 & 0.07 \\
\hline 54558.83 & 2008 Apr 2.83 & $r^{\prime}$ & 18.90 & 0.06 \\
\hline 54560.83 & 2008 Apr 4.83 & $r^{\prime}$ & 18.84 & 0.07 \\
\hline 54561.83 & 2008 Apr 5.83 & $r^{\prime}$ & 18.91 & 0.07 \\
\hline 54562.83 & 2008 Apr 6.83 & $r^{\prime}$ & 18.92 & 0.07 \\
\hline 54563.83 & 2008 Apr 7.83 & $r^{\prime}$ & 18.94 & 0.06 \\
\hline 54564.83 & 2008 Apr 8.83 & $r^{\prime}$ & 18.87 & 0.07 \\
\hline 54566.83 & 2008 Apr 10.83 & $r^{\prime}$ & 19.01 & 0.06 \\
\hline 54568.83 & 2008 Apr 12.83 & $r^{\prime}$ & 19.03 & 0.09 \\
\hline 54570.83 & 2008 Apr 14.83 & $r^{\prime}$ & 19.19 & 0.16 \\
\hline 54580.83 & 2008 Apr 24.83 & $r^{\prime}$ & 18.82 & 0.12 \\
\hline 54582.83 & 2008 Apr 26.83 & $r^{\prime}$ & 19.23 & 0.12 \\
\hline 54584.83 & 2008 Apr 28.83 & $r^{\prime}$ & 18.97 & 0.12 \\
\hline 54588.24 & 2008 May 2.24 & $r^{\prime}$ & 19.27 & 0.08 \\
\hline 54590.24 & 2008 May 4.24 & $r^{\prime}$ & 19.17 & 0.08 \\
\hline 54593.24 & 2008 May 7.24 & $r^{\prime}$ & 19.13 & 0.07 \\
\hline 54594.24 & 2008 May 8.24 & $r^{\prime}$ & 19.14 & 0.07 \\
\hline 54596.24 & 2008 May 10.24 & $r^{\prime}$ & 19.16 & 0.12 \\
\hline 54598.24 & 2008 May 12.24 & $r^{\prime}$ & 19.28 & 0.14 \\
\hline 54615.24 & 2008 May 29.24 & $r^{\prime}$ & 19.32 & 0.12 \\
\hline 54617.24 & 2008 May 31.24 & $r^{\prime}$ & 19.20 & 0.26 \\
\hline 54618.65 & 2008 Jun 1.65 & $r^{\prime}$ & 19.35 & 0.10 \\
\hline 54619.65 & 2008 Jun 2.65 & $r^{\prime}$ & 19.61 & 0.12 \\
\hline 54624.65 & 2008 Jun 7.65 & $r^{\prime}$ & 19.43 & 0.10 \\
\hline 54628.65 & 2008 Jun 11.65 & $r^{\prime}$ & 19.62 & 0.18 \\
\hline 54636.65 & 2008 Jun 19.65 & $r^{\prime}$ & 18.94 & 0.16 \\
\hline 54638.65 & 2008 Jun 21.65 & $r^{\prime}$ & 19.37 & 0.19 \\
\hline 54640.65 & 2008 Jun 23.65 & $r^{\prime}$ & 19.19 & 0.09 \\
\hline 54642.65 & 2008 Jun 25.65 & $r^{\prime}$ & 19.16 & 0.10 \\
\hline 54645.65 & 2008 Jun 28.65 & $r^{\prime}$ & 18.90 & 0.10 \\
\hline 54648.06 & $2008 \mathrm{Jul} 1.06$ & $r^{\prime}$ & 19.22 & 0.11 \\
\hline 54787.72 & 2008 Nov 1.72 & $r^{\prime}$ & 19.33 & 0.17 \\
\hline 54800.71 & 2008 Nov 3.71 & $r^{\prime}$ & 19.57 & 0.17 \\
\hline 54805.13 & 2008 Dec 5.13 & $r^{\prime}$ & 19.57 & 0.11 \\
\hline 54811.13 & 2008 Dec 1.13 & $r^{\prime}$ & 19.51 & 0.14 \\
\hline 54836.54 & 2009 Jan 5.54 & $r^{\prime}$ & 19.83 & 0.13 \\
\hline 54496.54 & 2008 Jan 31.54 & $i^{\prime}$ & 18.30 & 0.08 \\
\hline 54496.54 & 2008 Jan 31.54 & $i^{\prime}$ & 18.36 & 0.07 \\
\hline 54505.95 & 2008 Feb 9.95 & $i^{\prime}$ & 18.42 & 0.07 \\
\hline 54508.95 & 2008 Feb 12.95 & $i^{\prime}$ & 18.49 & 0.07 \\
\hline 54509.95 & 2008 Feb 13.95 & $i^{\prime}$ & 18.38 & 0.06 \\
\hline 54513.95 & 2008 Feb 17.95 & $i^{\prime}$ & 18.44 & 0.06 \\
\hline 54514.95 & 2008 Feb 18.95 & $i^{\prime}$ & 18.44 & 0.14 \\
\hline 54515.95 & 2008 Feb 19.95 & $i^{\prime}$ & 18.53 & 0.11 \\
\hline 54522.95 & 2008 Feb 26.95 & $i^{\prime}$ & 18.65 & 0.07 \\
\hline 54523.95 & 2008 Feb 27.95 & $i^{\prime}$ & 18.30 & 0.07 \\
\hline
\end{tabular}


Table 4

(Continued)

\begin{tabular}{|c|c|c|c|c|}
\hline $\mathrm{MJD}^{\mathrm{a}}$ & UT Date & Filter & Magnitude $^{\mathrm{b}}$ & Error \\
\hline 54524.95 & 2008 Feb 28.95 & $i^{\prime}$ & 18.43 & 0.06 \\
\hline 54525.95 & 2008 Feb 29.95 & $i^{\prime}$ & 18.50 & 0.07 \\
\hline 54531.41 & 2008 Mar 6.41 & $i^{\prime}$ & 18.42 & 0.07 \\
\hline 54533.41 & 2008 Mar 8.41 & $i^{\prime}$ & 18.49 & 0.08 \\
\hline 54535.41 & 2008 Mar 10.41 & $i^{\prime}$ & 18.55 & 0.09 \\
\hline 54537.41 & 2008 Mar 12.41 & $i^{\prime}$ & 18.48 & 0.13 \\
\hline 54538.41 & 2008 Mar 13.41 & $i^{\prime}$ & 18.57 & 0.08 \\
\hline 54539.41 & 2008 Mar 14.41 & $i^{\prime}$ & 18.42 & 0.06 \\
\hline 54543.41 & 2008 Mar 18.41 & $i^{\prime}$ & 18.64 & 0.11 \\
\hline 54544.41 & 2008 Mar 19.41 & $i^{\prime}$ & 18.58 & 0.10 \\
\hline 54545.41 & 2008 Mar 20.41 & $i^{\prime}$ & 18.46 & 0.15 \\
\hline 54550.41 & 2008 Mar 25.41 & $i^{\prime}$ & 18.59 & 0.09 \\
\hline 54551.41 & 2008 Mar 26.41 & $i^{\prime}$ & 18.66 & 0.07 \\
\hline 54554.41 & 2008 Mar 29.41 & $i^{\prime}$ & 18.52 & 0.06 \\
\hline 54556.41 & 2008 Mar 31.41 & $i^{\prime}$ & 18.67 & 0.07 \\
\hline 54556.41 & 2008 Mar 31.41 & $i^{\prime}$ & 18.74 & 0.08 \\
\hline 54557.83 & 2008 Apr 1.83 & $i^{\prime}$ & 18.64 & 0.08 \\
\hline 54558.83 & 2008 Apr 2.83 & $i^{\prime}$ & 18.50 & 0.11 \\
\hline 54560.83 & 2008 Apr 4.83 & $i^{\prime}$ & 18.62 & 0.07 \\
\hline 54561.83 & 2008 Apr 5.83 & $i^{\prime}$ & 18.58 & 0.08 \\
\hline 54562.83 & 2008 Apr 6.83 & $i^{\prime}$ & 18.78 & 0.08 \\
\hline 54563.83 & 2008 Apr 7.83 & $i^{\prime}$ & 18.55 & 0.06 \\
\hline 54564.83 & 2008 Apr 8.83 & $i^{\prime}$ & 18.59 & 0.08 \\
\hline 54566.83 & 2008 Apr 10.83 & $i^{\prime}$ & 18.83 & 0.08 \\
\hline 54568.83 & 2008 Apr 12.83 & $i^{\prime}$ & 18.70 & 0.08 \\
\hline 54570.83 & 2008 Apr 14.83 & $i^{\prime}$ & 18.63 & 0.15 \\
\hline 54577.83 & 2008 Apr 21.83 & $i^{\prime}$ & 18.93 & 0.18 \\
\hline 54578.83 & 2008 Apr 22.83 & $i^{\prime}$ & 19.26 & 0.23 \\
\hline 54580.83 & 2008 Apr 24.83 & $i^{\prime}$ & 18.61 & 0.09 \\
\hline 54582.83 & 2008 Apr 26.83 & $i^{\prime}$ & 18.70 & 0.10 \\
\hline 54584.83 & 2008 Apr 28.83 & $i^{\prime}$ & 18.85 & 0.14 \\
\hline 54588.24 & 2008 May 2.24 & $i^{\prime}$ & 18.76 & 0.08 \\
\hline 54590.24 & 2008 May 4.24 & $i^{\prime}$ & 18.71 & 0.08 \\
\hline 54593.24 & 2008 May 7.24 & $i^{\prime}$ & 18.68 & 0.07 \\
\hline 54594.24 & 2008 May 8.24 & $i^{\prime}$ & 18.79 & 0.10 \\
\hline 54596.24 & 2008 May 10.24 & $i^{\prime}$ & 18.82 & 0.13 \\
\hline 54598.24 & 2008 May 12.24 & $i^{\prime}$ & 18.84 & 0.12 \\
\hline 54600.24 & 2008 May 14.24 & $i^{\prime}$ & 18.93 & 0.16 \\
\hline 54606.24 & 2008 May 20.24 & $i^{\prime}$ & 19.06 & 0.16 \\
\hline 54615.24 & 2008 May 29.24 & $i^{\prime}$ & 18.57 & 0.10 \\
\hline 54634.65 & 2008 Jun 17.65 & $i^{\prime}$ & 18.81 & 0.13 \\
\hline 54636.65 & 2008 Jun 19.65 & $i^{\prime}$ & 18.81 & 0.11 \\
\hline 54640.65 & 2008 Jun 23.65 & $i^{\prime}$ & 18.94 & 0.09 \\
\hline 54642.65 & 2008 Jun 25.65 & $i^{\prime}$ & 18.74 & 0.08 \\
\hline 54645.65 & 2008 Jun 28.65 & $i^{\prime}$ & 19.18 & 0.14 \\
\hline 54648.06 & 2008 Jul 1.06 & $i^{\prime}$ & 18.95 & 0.12 \\
\hline 54655.06 & $2008 \mathrm{Jul} 8.06$ & $i^{\prime}$ & 18.95 & 0.15 \\
\hline 54800.71 & 2008 Nov 3.71 & $i^{\prime}$ & 19.32 & 0.15 \\
\hline 54496.54 & 2008 Jan 31.54 & $z^{\prime}$ & 18.18 & 0.14 \\
\hline 54505.95 & 2008 Feb 9.95 & $z^{\prime}$ & 18.74 & 0.18 \\
\hline 54508.95 & 2008 Feb 12.95 & $z^{\prime}$ & 18.55 & 0.12 \\
\hline 54523.95 & 2008 Feb 27.95 & $z^{\prime}$ & 17.87 & 0.12 \\
\hline 54524.95 & 2008 Feb 28.95 & $z^{\prime}$ & 18.29 & 0.14 \\
\hline 54525.95 & 2008 Feb 29.95 & $z^{\prime}$ & 18.25 & 0.12 \\
\hline 54531.41 & 2008 Mar 6.41 & $z^{\prime}$ & 18.35 & 0.22 \\
\hline 54533.41 & 2008 Mar 8.41 & $z^{\prime}$ & 18.36 & 0.16 \\
\hline 54534.41 & 2008 Mar 9.41 & $z^{\prime}$ & 18.20 & 0.13 \\
\hline 54536.41 & 2008 Mar 11.41 & $z^{\prime}$ & 18.26 & 0.11 \\
\hline 54537.41 & 2008 Mar 12.41 & $z^{\prime}$ & 18.74 & 0.22 \\
\hline 54539.41 & 2008 Mar 14.41 & $z^{\prime}$ & 18.52 & 0.14 \\
\hline 54543.41 & 2008 Mar 18.41 & $z^{\prime}$ & 18.05 & 0.13 \\
\hline 54544.41 & 2008 Mar 19.41 & $z^{\prime}$ & 18.30 & 0.18 \\
\hline 54549.41 & 2008 Mar 24.41 & $z^{\prime}$ & 18.29 & 0.20 \\
\hline 54550.41 & 2008 Mar 25.41 & $z^{\prime}$ & 18.35 & 0.12 \\
\hline 54551.41 & 2008 Mar 26.41 & $z^{\prime}$ & 18.52 & 0.12 \\
\hline 54557.83 & 2008 Apr 1.83 & $z^{\prime}$ & 18.25 & 0.15 \\
\hline
\end{tabular}

Table 4

(Continued)

\begin{tabular}{llccc}
\hline \hline MJD $^{\mathrm{a}}$ & UT Date & Filter & Magnitude $^{\mathrm{b}}$ & Error \\
\hline 54560.83 & 2008 Apr 4.83 & $z^{\prime}$ & 18.14 & 0.14 \\
54566.83 & 2008 Apr 10.83 & $z^{\prime}$ & 18.86 & 0.23 \\
54570.83 & 2008 Apr 14.83 & $z^{\prime}$ & 17.92 & 0.32 \\
54577.83 & 2008 Apr 21.83 & $z^{\prime}$ & 18.03 & 0.17 \\
54578.83 & 2008 Apr 22.83 & $z^{\prime}$ & 18.02 & 0.22 \\
54584.83 & 2008 Apr 28.83 & $z^{\prime}$ & 18.00 & 0.16 \\
54588.24 & 2008 May 2.24 & $z^{\prime}$ & 18.72 & 0.23 \\
54594.24 & 2008 May 8.24 & $z^{\prime}$ & 18.15 & 0.23 \\
54596.24 & 2008 May 10.24 & $z^{\prime}$ & 18.37 & 0.17 \\
54603.24 & 2008 May 17.24 & $z^{\prime}$ & 18.22 & 0.25 \\
54606.24 & 2008 May 20.24 & $z^{\prime}$ & 18.70 & 0.22 \\
54640.65 & 2008 Jun 23.65 & $z^{\prime}$ & 18.49 & 0.13 \\
54655.06 & 2008 Jul 8.06 & $z^{\prime}$ & 17.99 & 0.26 \\
54787.17 & 2008 Nov 17.17 & $z^{\prime}$ & 17.71 & 0.24 \\
\hline
\end{tabular}

Notes.

a MJD values refer to the observer frame.

${ }^{\mathrm{b}}$ Corrected for extinction.

Table 5

Swift UVOT Photometry of SN 2008am

\begin{tabular}{|c|c|c|c|c|}
\hline$\overline{\mathrm{MJD}^{\mathrm{a}}}$ & UT Date & Filter & Magnitude $^{\mathrm{b}}$ & Error \\
\hline 54502.95 & 2008 Feb 7.95 & V & 18.96 & 0.30 \\
\hline 54503.95 & 2008 Feb 8.95 & $V$ & 18.37 & 0.23 \\
\hline 54511.95 & 2008 Feb 16.95 & $V$ & 19.45 & 0.52 \\
\hline 54575.83 & 2008 Apr 19.83 & $V$ & 19.59 & 0.32 \\
\hline 54502.95 & 2008 Feb 7.95 & $B$ & 19.19 & 0.14 \\
\hline 54511.95 & 2008 Feb 16.95 & $B$ & 19.47 & 0.23 \\
\hline 54575.83 & 2008 Apr 19.83 & $B$ & 19.97 & 0.19 \\
\hline 54502.95 & 2008 Feb 7.95 & $U$ & 18.15 & 0.09 \\
\hline 54509.95 & 2008 Feb 14.95 & $U$ & 18.54 & 0.19 \\
\hline 54511.95 & 2008 Feb 16.95 & $U$ & 18.49 & 0.14 \\
\hline 54575.83 & 2008 Apr 19.83 & $U$ & 19.40 & 0.16 \\
\hline 54502.95 & 2008 Feb 7.95 & $U V W 1$ & 18.51 & 0.10 \\
\hline 54503.95 & 2008 Feb 8.95 & $U V W 1$ & 18.26 & 0.34 \\
\hline 54509.95 & 2008 Feb 14.95 & $U V W 1$ & 18.53 & 0.17 \\
\hline 54511.95 & 2008 Feb 16.95 & $U V W 1$ & 18.86 & 0.16 \\
\hline 54538.41 & 2008 Mar 14.41 & $U V W 1$ & 19.33 & 0.13 \\
\hline 54575.83 & 2008 Apr 19.83 & $U V W 1$ & 19.63 & 0.15 \\
\hline 54502.95 & 2008 Feb 7.95 & $U V M 2$ & 18.52 & 0.16 \\
\hline 54503.95 & 2008 Feb 8.95 & $U V M 2$ & 18.45 & 0.24 \\
\hline 54511.95 & 2008 Feb 16.95 & $U V M 2$ & 18.66 & 0.15 \\
\hline 54538.41 & 2008 Mar 14.41 & $U V M 2$ & 19.18 & 0.12 \\
\hline 54575.83 & 2008 Apr 19.83 & $U V M 2$ & 19.26 & 0.13 \\
\hline 54502.95 & 2008 Feb 7.95 & $U V W 2$ & 18.78 & 0.08 \\
\hline 54503.95 & 2008 Feb 8.95 & $U V W 2$ & 18.77 & 0.22 \\
\hline 54511.95 & 2008 Feb 16.95 & $U V W 2$ & 19.08 & 0.12 \\
\hline 54538.41 & 2008 Mar 14.41 & $U V W 2$ & 19.64 & 0.12 \\
\hline 54575.83 & 2008 Apr 19.83 & $U V W 2$ & 19.51 & 0.09 \\
\hline
\end{tabular}

Notes.

${ }^{a}$ MJD values refer to the observer frame.

${ }^{\mathrm{b}}$ Observed value; not corrected for extinction.

The rest-frame light curves of SN 2008am for all the available photometric bands are shown in Figure 4. The top left panel shows the PAIRITEL IR J and $\mathrm{H}$ light curves, the top right panel the Swift UVOT optical and UV light curves and the bottom panel the ROTSE unfiltered and P60 optical light curves. The data sets have been offset for clarity, with longer wavelength on top and shorter wavelength toward the bottom of each panel. The broad photometric wavelength coverage allows us to better constrain the SED of the SN. 

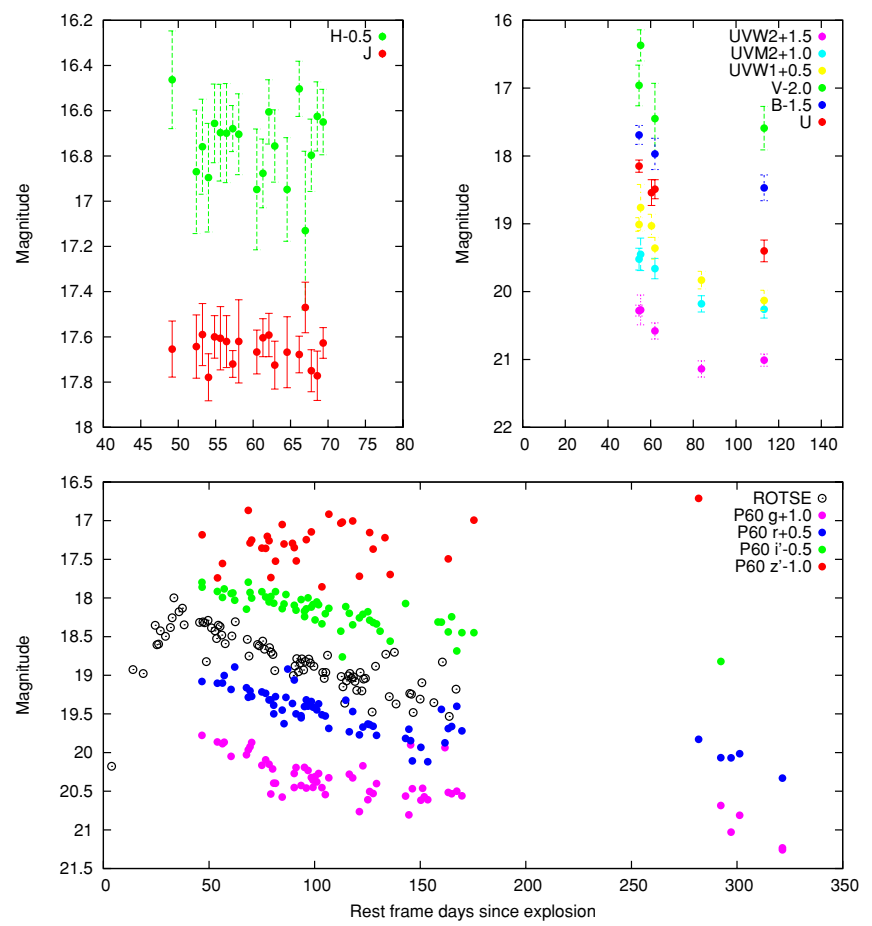

Figure 4. Rest-frame light curves of SN 2008am from the IR to the UV. Top left panel: the PAIRITEL IR $J$ - and $H$-band light curves. Top right panel: the Swift UVOT optical and UV light curves. Bottom panel: the ROTSE unfiltered (open circles) and the optical P60 (filled circles) light curves. The data have been offset for clarity.

(A color version of this figure is available in the online journal.)

SN 2008am was observed with the Very Large Array at $8.46 \mathrm{GHz}$ on 2008 February $19.37 \mathrm{UT}$, approximately 30 restframe days after optical maximum (Chandra \& Soderberg 2008). No source was detected at the SN position above $120 \mu \mathrm{Jy}$, which can be considered as a $3 \sigma$ upper limit. Swift X-ray Telescope (XRT; Burrows et al. 2005) images of SN 2008am were obtained for the six epochs in parallel with the UVOT observations. No $\mathrm{X}$-ray source was detected at the position of the SN, after coadding all XRT observations. Assuming a power-law spectrum with spectral index of -2 , the X-ray flux upper limit was estimated to be $\sim 10^{-13} \mathrm{erg} \mathrm{cm}^{-2} \mathrm{~s}^{-1}$ (or, equivalently $\sim 10^{43} \mathrm{erg} \mathrm{s}^{-1}$ ) at $\sim 50$ rest-frame days since explosion.

\subsection{The SED of SN 2008 am}

The availability of multi-band simultaneous photometry for some epochs helps us construct SEDs for SN 2008am and thus study the evolution and basic properties of the event. Our criteria for selecting the photometric epochs for which we constructed the SEDs were two: close sampling in time and maximum possible wavelength coverage. Those criteria led to the choice of 10 epochs for which we constructed the photometric SEDs. For four of those epochs we had available UV+Optical+IR observations (hereafter UVOIR), for four only Optical (P60 data, hereafter OPT) and for two (08-01-30 and 08-02-25) Optical+IR (see Figure 4).

For the 08-02-25 epoch, we interpolated between the previous (08-02-16) and the next (08-03-14) epoch for which we had UV data. The error of this interpolated value was estimated using error propagation analysis for a linear fit. We note that the UV flux for the interpolated 08-02-25 epoch is uncertain since the UV light curve decline of emission line objects like SN 2008am is not well constrained at later times. Adding the interpolated

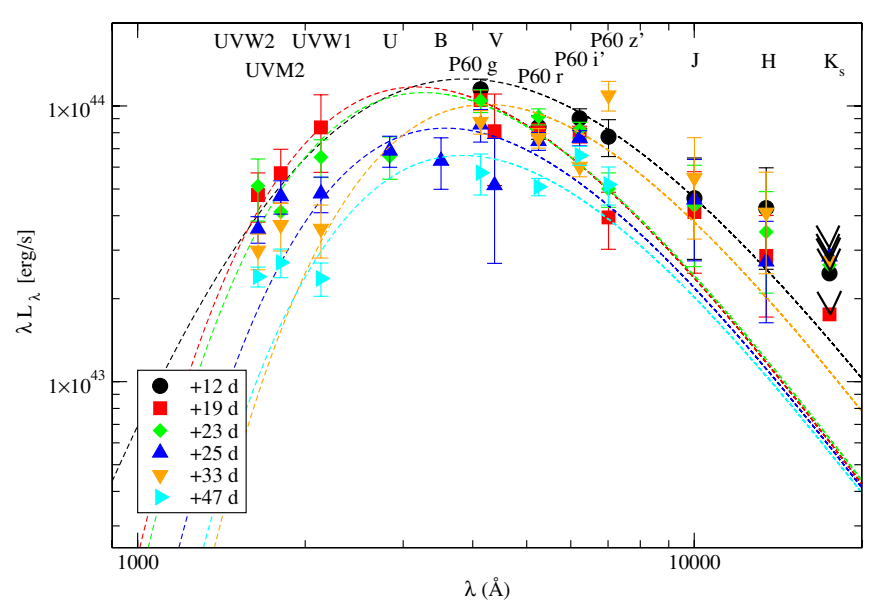

Figure 5. Six (five UVOIR plus one OIR) SEDs of SN 2008am and their corresponding single-temperature blackbody fits. The black arrows in the restframe $K$-band fluxes indicate upper limits. All the phases in the inset refer to the rest-frame time in days since maximum. The photometric filters at their redshifted peak wavelength position are also indicated.

(A color version of this figure is available in the online journal.)

UV data to the Optical+IR data gave us a total of five epochs of UVOIR data and one epoch (08-01-30) for OIR data that we will later use to estimate the pseudo-bolometric light curve (LC) of the event.

Although we see no sign of classic supernova photospheric P Cygni lines (see Sections 2.4 and 3), the blue continua of the spectra are consistent with thermal emission. This emission could arise in shocked CSM that is modestly optically thick $(\tau \sim 1)$ to absorption. To produce a quantitative diagnostic, we fit single-temperature blackbody curves to the set of the five UVOIR and one OIR photometric SEDs in order to determine effective blackbody temperatures in the rest frame. We included the reddening corrections discussed in Section 2.2. The blackbody curve fits were done in the rest frame of the SN. Bolometric luminosities were then derived from the integral of the flux from the corresponding blackbody at the adopted distance of the supernova. Effective blackbody radii were derived from the luminosity and blackbody temperature. We did not attempt to fit the OPT epochs with single-temperature blackbody curves as the uncertainties of the fit would be large, given the lack of UV data. We estimated lower limits for the bolometric luminosity in these four epochs by integrating the observed SED.

Figure 5 shows the photometric SEDs of SN 2008am for the six selected epochs together with the best-fit (lowest $\chi^{2}$ ) blackbody curve in each case. Figure 6 shows two examples of UVOIR SEDs and their blackbody curve fits and nearly contemporaneous spectral continua at +22 days and +33 days after maximum, respectively. Blackbody fits to the spectroscopic data were also done in the rest frame and included the reddening corrections of Section 2.2. The spectra of SN 2008am were scaled to the simultaneous photometry in each case, before any reddening corrections were applied. The temperatures derived from the spectral fits agree with those derived from the fits to the SEDs within the errors at these two epochs (Figure 6).

Table 6 summarizes the characteristics of the blackbody fits to the UVOIR and OIR SEDs of SN 2008am, the effective blackbody temperature, $T_{\mathrm{bb}}$, the effective blackbody radius, $R_{\mathrm{bb}}$, and the derived bolometric luminosity, $L_{\mathrm{bol}}$. Figure 7 presents the evolution of $T_{\mathrm{bb}}$ (upper panel), $R_{\mathrm{bb}}$ (middle panel), and $L_{\mathrm{bol}}$ 
Table 6

Characteristics of the Blackbody Fits to the Photometric SEDs of SN 2008am

\begin{tabular}{lccccc}
\hline \hline Epoch (MJD) & $t_{\mathrm{rf}}($ days $)$ & $\chi^{2} / \mathrm{dof}$ & $T_{\mathrm{bb}}\left(10^{4} \mathrm{~K}\right)$ & $R_{\mathrm{bb}}\left(10^{15} \mathrm{~cm}\right)$ & $L_{\mathrm{bol}}\left(10^{44} \mathrm{erg} \mathrm{s}-1\right)$ \\
\hline 54495 & +12 & 2.4 & $0.910(0.140)$ & $5.530(0.960)$ & $1.490(0.410)$ \\
54504 & +19 & 1.3 & $1.200(0.060)$ & $3.190(0.220)$ & $1.420(0.100)$ \\
54509 & +23 & 2.7 & $1.020(0.180)$ & $4.020(0.840)$ & $1.230(0.220)$ \\
54512 & +25 & 2.4 & $1.200(0.170)$ & $2.520(0.430)$ & $0.950(0.150)$ \\
54521 & +33 & 2.9 & $1.020(0.150)$ & $3.930(0.840)$ & $1.180(0.260)$ \\
54539 & +47 & 3.7 & $1.050(0.230)$ & $3.030(0.910)$ & $0.790(0.250)$ \\
\hline
\end{tabular}

Notes. The numbers in parentheses represent the estimated error of each parameter. The $t_{\mathrm{rf}}$ column refers to rest-frame days since maximum.

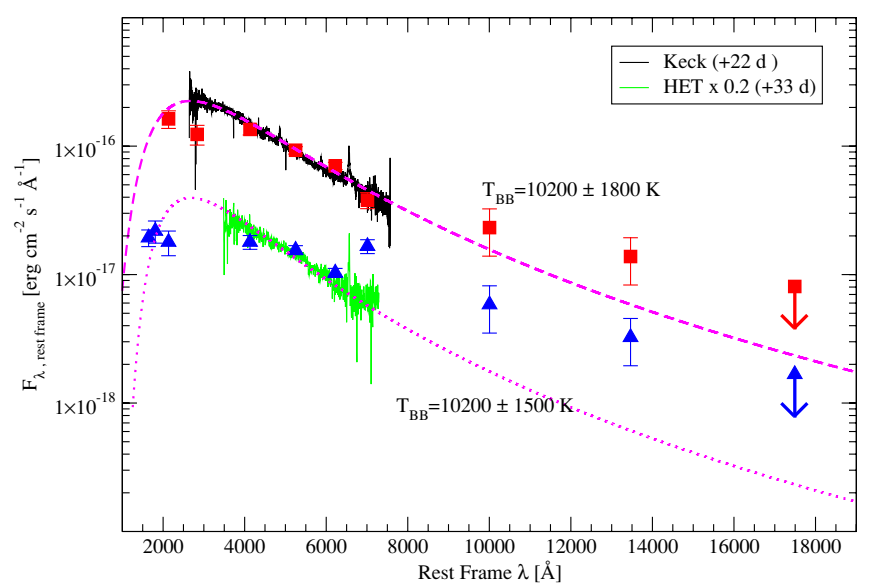

Figure 6. Blackbody curve fits for SN 2008am for two epochs for which there is photometric and spectroscopic overlap. The photometric SED for rest-frame day +23 (red squares) is constructed using UVOT+P60+PAIRITEL $J$ data and the SED for rest-frame day +33 (blue triangles) uses P60+PAIRITEL data. The best-fit blackbody curves for the photometric data are given by the dashed line $(+23 \mathrm{~d})$ and the dotted line $(+33 \mathrm{~d})$. The corresponding temperatures (see Table 6) are given next to the respective blackbody curves. The temperatures of the blackbody fits to the spectra are $11,100 \mathrm{~K}$ on day +22 and $12,200 \mathrm{~K}$ on day +33 . The HET LRS spectrum has been scaled down by a factor of five for presentation.

(A color version of this figure is available in the online journal.)

(lower panel). The filled triangles correspond to the fits to the UVOIR and OIR SEDs and the open circles to those of four early Hobby-Eberly Telescope (HET) and Keck spectra (the fifth, obtained on 2008 January 30.3 has a slightly anomalous slope that we attribute to observing conditions; Section 2.4). The single-temperature blackbody fits to the SEDs of SN 2008am show considerable uncertainty and scatter. The photometric points scatter around the best-fit blackbody curves and around the spectral continua. Differences between the spectral and photometric results at similar epochs may be attributed to the fact that the optical spectra do not accurately constrain the maximum of the blackbody curve and that we assumed a single blackbody to fit the SEDs and not more complex models. At the latest epochs, there could be other effects (for example, dust formation) that affect the final estimates. The NIR data are always in excess with respect to the fitted curves. That may be an indication that the single-temperature blackbody models do not accurately represent the emission properties of SN 2008am, but it is also possible that the NIR data are still somewhat contaminated by the host galaxy and only represent upper limits to the corresponding supernova flux. Another possibility is that the NIR excess is a sign of early warm dust emission (Meikle et al. 2007; Mattila et al. 2008; Kotak et al. 2009; Fox et al. 2010). The IR data are thus especially uncertain, but these data

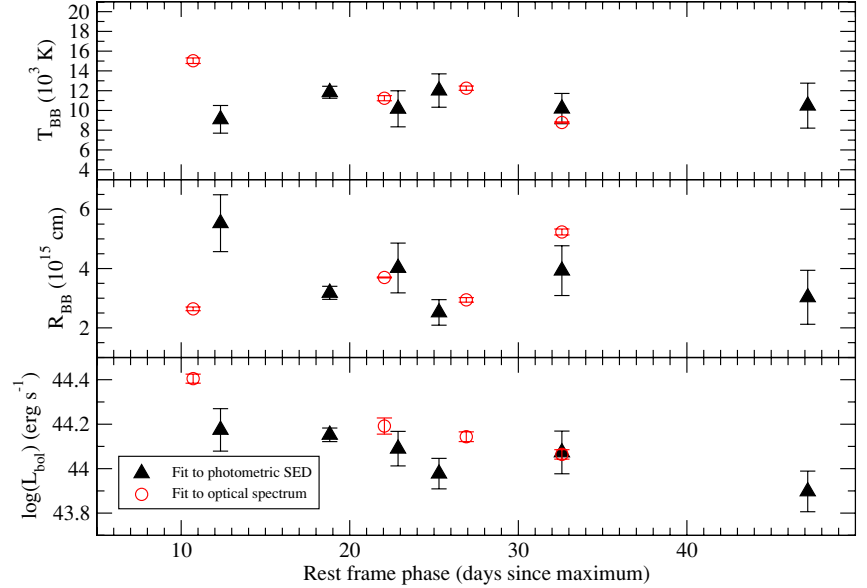

Figure 7. Evolution of the effective blackbody temperature $T_{\mathrm{bb}}$ (upper panel), the effective blackbody radius $R_{\mathrm{bb}}$ (middle panel), and the bolometric luminosity $L_{\text {bol }}$ (lower panel) of SN 2008am as estimated by blackbody fits to the restframe photometric SEDs for the six epochs for which we have UVOIR and OIR data. The filled triangles refer to the fits to the photometric SEDs. The open circles represent blackbody fits to optical spectra at similar phases.

(A color version of this figure is available in the online journal.)

have relatively little weight in the blackbody fits. The blackbody temperatures and radii derived here are only indicative of the general conditions and their trends, and not to be treated as quantitatively precise nor as evidence that the emission is truly blackbody.

The effective blackbody temperatures are in the range of 10,000-12,000 K, as presented in Table 6 and Figure 7, and are constant in time within the scatter. The pseudo-bolometric light curve implies a total radiated energy of about $10^{51} \mathrm{erg}$. Other estimates of the total radiated energy will be given in Section 4 based on several physical models. We will argue in Section 4.4 that the energy powering SN 2008am is most probably the interaction between the ejecta and the CSM and thus the estimates of $T_{\mathrm{bb}}, R_{\mathrm{bb}}$, and $L_{\mathrm{bol}}$ do not correspond to an expanding photosphere coincident with the ejecta of the SN. The ejecta of the SN and the CSM shocks may be hidden behind an optically thick CSM. In this case, the values of $T_{\mathrm{bb}}, R_{\mathrm{bb}}$, and $L_{\text {bol }}$ refer to conditions in an optically thick CSM with $\tau \sim 1$ representing the effective photosphere of the CSM.

\subsection{Spectroscopy}

We acquired a total of seven spectra of SN 2008am and its host galaxy spanning $\sim 2 \mathrm{yr}$ after discovery. Four of them were taken with the HET Low Resolution Spectrograph (LRS; Hill et al. 1998) on 2008 January 29.3 UT, 2008 January 30.3, 2008 February 18.3, and 2008 February 25.3, corresponding 


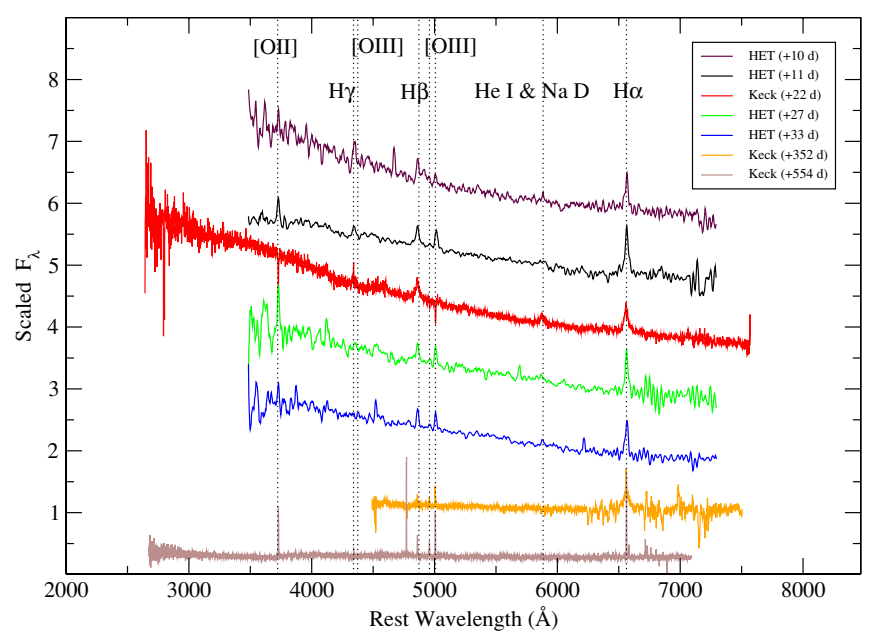

Figure 8. Spectral evolution of SN 2008am in the rest frame. The phases given in the inset refer to the rest-frame time in days since maximum. The dotted vertical lines mark the positions of the $\mathrm{H}, \mathrm{He}$, and $\mathrm{O}$ features. Scaling and offsets to the flux values of the original spectra have been applied for clarity.

(A color version of this figure is available in the online journal.)

to $+11,+12,+27$, and +33 rest-frame days after maximum, respectively. Two other spectra were obtained with the KeckI Low Resolution Imaging Spectrograph (LRIS; Oke et al. 1995) on 2008 February 12.0 UT (+22 days after maximum in rest frame) and 2009 March 31.0 (+352 rest-frame days after maximum). Another Keck-LRIS spectrum of the host galaxy was taken on 2010 January 09.0 UT, +554 rest-frame days after maximum, when the transient had faded below the detection limit. All spectra were reduced in the standard way using IRAF. The instrumental resolution was $\sim 17 \AA$ for the HET spectra and $\sim 6 \AA$ for the Keck spectra.

We will refer to the spectra obtained within a month after maximum as "early-phase" and the +352 day Keck spectrum as "late-phase," respectively. All spectra were corrected for Milky Way reddening (as described in Section 2.2) and scaled to contemporaneous photometric data. The epochs of the early spectroscopic observations relative to the light curve are shown in Figure 3.

Figure 8 shows the spectral evolution of SN 2008am. Note that the first HET spectrum (obtained on 2008 January 29.3) is plotted for completeness, but omitted from further analysis because of the availability of the second spectrum (taken one day later, on January 30.3) that was obtained during better observing conditions giving better signal-to-noise ratio $(\mathrm{S} / \mathrm{N})$. The January 30.3 spectrum has a somewhat discrepant slope for reasons we have been unable to resolve, but the continuum slope of the spectra does not enter into our analysis except for perhaps a very small effect on line profiles. The 2008 January 29.3 spectrum was used in the blackbody fits (Section 2.3; Figure 7).

The spectra were deredshifted by $z=0.2338$ determined from the narrow emission features in the host spectrum. This value is very close to the one derived by Yuan et al. (2008a) based on the early-phase HET spectra.

The early-phase spectra show prominent features of $\mathrm{H}(\mathrm{H} \alpha$, $\mathrm{H} \beta$, and $\mathrm{H} \gamma)$. The He $5876 \AA$ line is detected in the February 12 Keck spectrum and with a smaller $\mathrm{S} / \mathrm{N}$ in the February 25 HET spectrum. Na D may also contribute to this feature, but we were unable to make a definite identification.

The HET data had inferior seeing and lower spatial resolution of the spectrograph that made it impossible to fully resolve and separate the SN and the host. Given those instrumental
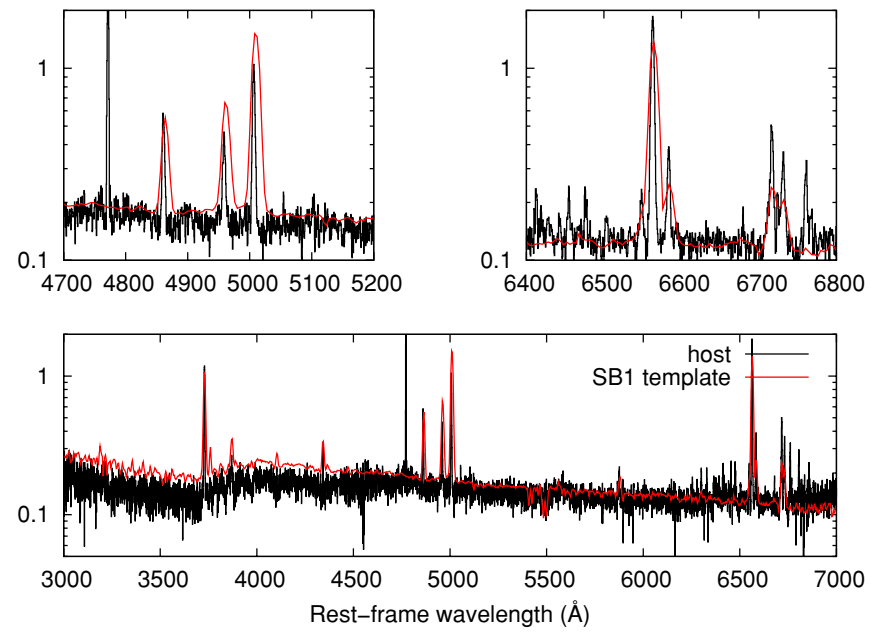

Figure 9. Late +554 day Keck spectrum of the host of SN 2008am (bottom panel; black solid curve) compared to an SB1 galaxy template (Kinney et al. 1996; red solid curve). The $\mathrm{H} \beta$ and [O III] region of the spectrum (top left). The $\mathrm{H} \alpha,[\mathrm{N} I \mathrm{I}]$, and [S II] region of the spectrum (top right).

(A color version of this figure is available in the online journal.)

differences between HET-LRS and Keck-LRIS we chose to analyze the spectra uncorrected for host extinction, so that we could treat all the data in a uniform way. The host probably contributes to some of the detected emission lines; [O II] $3727 \AA$ and [O III] $5007 \AA$ are undoubtedly present, and [O III] $4959 \AA$ can also be weakly detected in all HET spectra. Fortunately, the host contribution to the continuum should be much less, because the +554 day Keck spectrum, which is attributed to the host galaxy, shows mainly a flat, low continuum.

Due to better resolution and seeing, the host subtraction was possible for the early-phase and late-phase Keck spectra. The small visible spatial extension of the galaxy required a background region defined as close to the $\mathrm{SN}$ as possible to achieve good background subtraction. Most of the galactic forbidden emission lines have been removed successfully; however, this process resulted in a slight oversubtraction in the core of $\mathrm{H} \alpha$ for the early-phase Keck spectrum, forming an "absorption" dip on top of the line. That feature is due to the reduction process and should not be interpreted physically (see below). Although we show the host-subtracted early Keck spectrum for comparison, we note again that we do not use it for our analysis since host subtraction cannot be performed for the HET spectra given the different spatial resolution.

The late-phase Keck spectrum is also dominated by a broadened $\mathrm{H} \alpha$ line, but all other SN features have already faded below detectability. The shape of $\mathrm{H} \alpha$ strongly suggests that the transient was still visible at +352 days after maximum. A few narrow features at $\mathrm{H} \beta$ and around $\sim 5000 \AA$ also appear that are probably due to contribution from the host.

The host spectrum, obtained with Keck-LRIS at +554 restframe days after maximum (Figure 9), shows the usual narrow emission features of galaxies with ongoing star formation. Beside Balmer lines, we identified [O II] $3727 \AA$, [O III] 4959, $5007 \AA$, [N II] 6548, $6583 \AA$, and He I $5876 \AA$. There are also indications for the [S II] 6716, $6731 \AA$ features, but that region is heavily contaminated by tellurics, preventing a definite identification. All lines as well as the shape of the continuum (Figure 9; Section 2.1) can be very well matched by an SB1 galaxy template (Kinney et al. 1996). The metallicity of the host was estimated by computing the line flux ratios and the spectral indices N2 and O3N2 defined by Pettini \& Pagel (2004). 
Table 7

Summary of the $\mathrm{H} \alpha$ and $\mathrm{H} \beta$ Properties of SN 2008am

\begin{tabular}{|c|c|c|c|c|c|c|c|c|c|c|}
\hline Instrument $^{\mathrm{a}}$ & UT Date & Day $^{\mathrm{b}}$ & $\mathrm{EW}(\mathrm{H} \alpha)^{\mathrm{c}}$ & $\operatorname{FWHM}(\mathrm{H} \alpha)^{\mathrm{c}}$ & $\Delta \lambda_{0}(\mathrm{H} \alpha)^{\mathrm{c}}$ & $F(\mathrm{H} \alpha)^{\mathrm{c}}$ & $\mathrm{EW}(\mathrm{H} \beta)^{\mathrm{c}}$ & $\operatorname{FWHM}(\mathrm{H} \beta)^{\mathrm{c}}$ & $\Delta \lambda_{0}(\mathrm{H} \beta)^{\mathrm{c}}$ & $F(\mathrm{H} \beta)^{\mathrm{c}}$ \\
\hline HET-LRS & 2008 Jan 30.1 & +11 & -44 & 25 & 150 & 3.32 & -12 & 32 & 80 & 1.40 \\
\hline Keck-LRIS & 2008 Feb 12.0 & +22 & -27 & 39 & -4 & 1.67 & -11 & 41 & 102 & 1.07 \\
\hline Keck-LRIS $^{\mathrm{d}}$ & 2008 Feb 12.0 & +22 & -32 & 17 & 3 & 1.98 & -10 & 14 & 179 & 1.10 \\
\hline HET-LRS & $2008 \mathrm{Feb} 18.3$ & +27 & -48 & 22 & 85 & 2.55 & -11 & 27 & 67 & 1.17 \\
\hline HET-LRS & $2008 \mathrm{Feb} 25.3$ & +33 & -42 & 24 & 185 & 2.16 & -7 & 18 & 142 & 0.72 \\
\hline Keck-LRIS & 2009 Mar 31.0 & +352 & -307 & 25 & -60 & 1.05 & -3 & 3 & -72 & 0.06 \\
\hline
\end{tabular}

Notes.

a The HET-LRS and Keck-LRIS wavelength are 4020-10200 ̊̊ and 3500-8600 A, respectively.

${ }^{\mathrm{b}}$ All the values refer to the rest-frame days after maximum.

${ }^{c}$ The measured equivalent widths and fluxes refer to the rest-frame, galaxy-subtracted, and de-reddened spectra. The equivalent widths and FWHM are measured in $\AA$, the shifts of the line centers with respect to their rest-frame positions $\left(\Delta \lambda_{0}\right)$ in $\mathrm{km} \mathrm{s}^{-1}$, and fluxes in $10^{-15}$ erg cm${ }^{-2} \mathrm{~s}^{-1}$ computed by fitting Lorentzian profiles.

${ }^{\mathrm{d}}$ These estimates correspond to line profiles not corrected for host extinction.

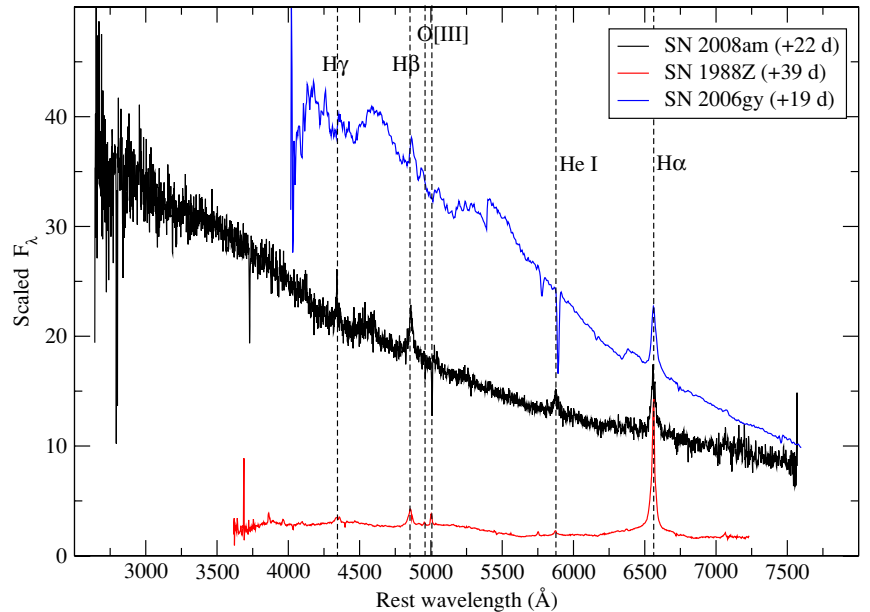

Figure 10. Comparison of the Keck spectrum of SN 2008am +22 days after maximum (rest frame) and the spectrum of the classical Type IIn supernova SN 1988Z in the rest frame +39 days after maximum (Stathakis \& Sadler 1991). The spectrum of the SLSN 2006gy at a similar rest-frame phase (+19 days after maximum) is also shown (Smith et al. 2010). The rest-frame positions of the emission lines of $\mathrm{H}, \mathrm{He}$ I (5876 $)$ ), and [O III] $\lambda \lambda$ 4959, 5007 are indicated with dashed vertical lines.

(A color version of this figure is available in the online journal.)

These resulted in an oxygen abundance of $12+\log (\mathrm{O} / \mathrm{H})=$ $8.38 \pm 0.15$, significantly below the solar abundance value $(\sim 8.7 \pm 0.1)$. This oxygen abundance suggests a sub-solar metallicity for the host, about $Z \sim 0.4 Z_{\odot}$. There is growing evidence that SLSNe, such as SN 2008am, occur mostly in metal-deficient hosts (Neill et al. 2011; Stoll et al. 2010).

None of the SN 2008am spectra show any sign of broad features characteristic of most $\mathrm{SNe}$ during the photospheric phase. There is no sign of P Cygni profiles. The Balmer lines in the early-phase spectra have FWHM $\sim 25 \AA$ in the rest frame, which are usually referred to as "intermediate-width" lines (e.g., Stathakis \& Sadler 1991). Such intermediate-width features are common characteristics of Type IIn SNe (e.g., Schlegel 1990). Based on these observed features, SN 2008am is certainly a member of the Type IIn subclass. The earlyphase spectra are similar to those of SN 1988Z (Stathakis \& Sadler 1991), SN 1995G (Pastorello et al. 2002), and the early spectra of SN 2006gy (Smith et al. 2010), all having blue continua with intermediate-width Balmer lines and without strong P Cygni profiles. In Figure 10, we plot the +22 day Keck
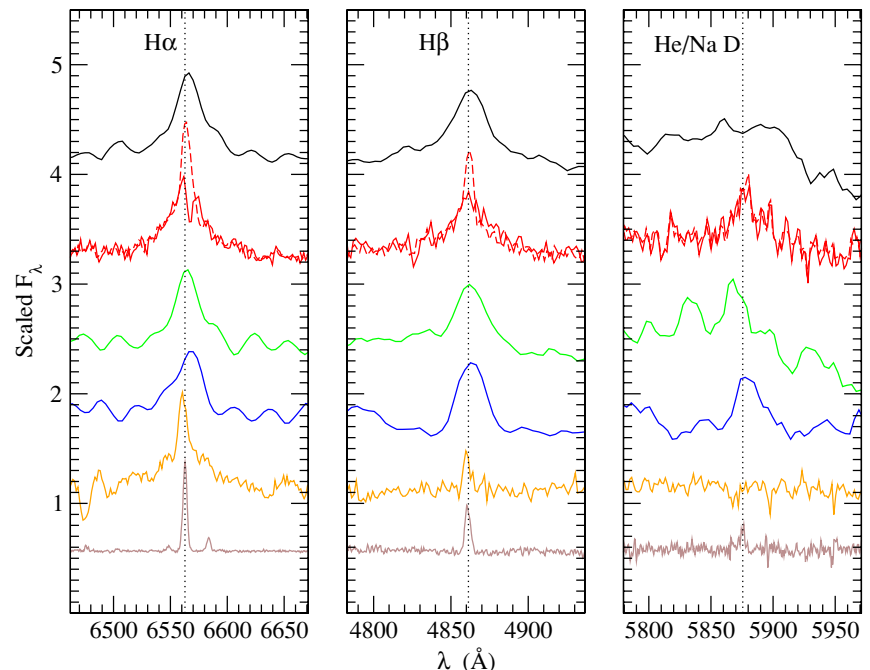

Figure 11. Evolution of the $\mathrm{H} \alpha$ (left panel), $\mathrm{H} \beta$ (middle panel), and $\mathrm{He} / \mathrm{Na} \mathrm{D}$ (right panel) line profiles of SN 2008am. The five spectra correspond to +12 days, +22 days, +27 days, +33 days, and +352 days from rest-frame maximum, respectively, from top to bottom. The final spectrum is of the host galaxy +554 rest-frame days after maximum. The vertical dotted lines show the restframe location of $\mathrm{H} \alpha, \mathrm{H} \beta$, and $\mathrm{He}$ I $\lambda$ 5876, respectively. For the Keck +22 day spectrum, we show the line profiles with (solid red curve) and without (dashed red curve) the effects of host subtraction.

(A color version of this figure is available in the online journal.)

spectrum together with the spectrum of SN $1998 \mathrm{Z}$ at +39 days after maximum light that had intermediate-width lines with no P Cygni components (Stathakis \& Sadler 1991; Turatto et al. 1993; Aretxaga et al. 1999). A spectrum of SN 2006gy taken 19 rest-frame days after maximum with the HET by one of us (R.Q.) is also shown in Figure 10 for comparison. Although some differences in the fine details exist, we argue below that SN 2008am and SN 2006gy exhibited remarkable similarities both in their spectral appearance and evolution.

Figure 11 shows the evolution of the emission lines that can be attributed to SN 2008am. For the early Keck spectrum, we give both the total flux profile (dashed red curve) and the line after host subtraction (solid red curve). The left panel presents $\mathrm{H} \alpha$, the middle panel $\mathrm{H} \beta$ and the right panel the $\mathrm{He} / \mathrm{Na} D$ feature that is only weakly detected. Dotted vertical lines mark the rest wavelength of the features. In Table 7, we list the basic parameters derived for $\mathrm{H} \alpha$ and $\mathrm{H} \beta$ : the shift of the line center with respect to its rest-frame position $\left(\Delta \lambda_{0}\right.$, 
expressed in $\mathrm{km} \mathrm{s}^{-1}$ ), the full width at half maximum (FWHM), integrated flux $(F)$, and equivalent width $(\mathrm{EW})$ based on fitting of Lorentzian profiles (more line profile models will be examined below).

In the earliest spectra, the line centers of both $\mathrm{H} \alpha$ and $\mathrm{H} \beta$ are redshifted with respect to their rest-frame position (determined from the narrow emission features of the host, as mentioned above). The average redshift is $115 \pm 40 \mathrm{~km} \mathrm{~s}^{-1}$ for both Balmer lines, consistent with some multiple-scattering models (Chugai 2001; Section 3). On the other hand, in the late-phase Keck spectrum the cores of the Balmer lines are slightly blueshifted by $-60 \mathrm{~km} \mathrm{~s}^{-1}$. The $\mathrm{H} \alpha$ profile in this spectrum looks narrower close to the peak, suggesting the presence of an unresolved narrow component, similar to the late-time $\mathrm{H} \alpha$ of SN 2006gy (Smith et al. 2010). This slight blueshift with respect to the restframe position may be explained by rotation of the host but might also arise from the effects of multiple scattering (Section 3).

The early-phase spectra have rest-frame FWHM line widths of $\sim 22 \AA$ and $\sim 23 \AA$ for $\mathrm{H} \alpha$ and $\mathrm{H} \beta$, respectively. Formally expressing these widths in terms of velocity (see Section 3 for a discussion on the effects of scattering), the FWHMs are $\sim 1000 \mathrm{~km} \mathrm{~s}^{-1}$ and $1400 \mathrm{~km} \mathrm{~s}^{-1}$ for $\mathrm{H} \alpha$ and $\mathrm{H} \beta$, respectively. $\mathrm{H} \beta$ seems to be broader in terms of velocity than $\mathrm{H} \alpha$. After removing the host flux, $\mathrm{H} \alpha$ appears broader in the early Keck spectrum than in the uncorrected spectrum or in any HET spectra, but a more detailed analysis showed that this is an artifact due to the depressed amplitude of the line core as a result of the host galaxy removal.

The HET spectra contain fluxes from both the SN and the host, thus the EWs and line fluxes are higher than those derived from the host-subtracted early-phase Keck spectrum. Comparing the numbers in Table 7 and correcting for the small host oversubtraction in the Keck spectrum, the host contribution to EWs and line fluxes in the HET spectra are estimated to be $\sim 17 \AA$ and $\sim 5 \times 10^{-16} \mathrm{erg} \mathrm{s}^{-1} \mathrm{~cm}^{-2}$, respectively.

The evolution of the line strengths and EWs for SN 2008am is very similar to that presented by Smith et al. (2010) for SN 2006gy and to several other Type IIn SNe. Shortly after maximum light the EW of $\mathrm{H} \alpha$ is $\sim 30-40 \AA$, rising to $\sim 300 \AA$ at late phases. The $\mathrm{H} \alpha$ integrated line fluxes, on the other hand, tend to decline in time. SNe are quite heterogeneous regarding the evolution of this parameter according to Smith et al. (2010), but the majority show a similar decline to that of SN 2008am. The line fluxes of $\mathrm{H} \beta$ also show a declining trend toward later phases, but the $\mathrm{EW}$ of $\mathrm{H} \beta$ is roughly constant with time.

During the early phase, the $\mathrm{F}(\mathrm{H} \alpha) / \mathrm{F}(\mathrm{H} \beta)$ ratio is $\sim 2.3 \pm 0.6$, which is close to the expected value in case $\mathrm{B}$ recombination (Osterbrock, 1989). SN 2006gy showed a very similar flux ratio close to maximum light (Smith et al. 2010). This ratio increases up to $\sim 17$ during the late phases in SN 2008am. This is also in accord with the observations of other SNe. An even higher flux ratio ( 30) was observed for SN 2006gy (Smith et al. 2010) and SN 2006tf (Smith et al. 2008) as well as for the strongly interacting Type IIn SN $1988 Z$ (Turatto et al. 1993).

The He I $5876 \AA$ line clearly shows up in the early-phase Keck spectrum and is also weakly detected in the HET spectra, although the latter data are noisy. The rest-frame FWHM of this line is formally measured to be $1800 \pm 600 \mathrm{~km} \mathrm{~s}^{-1}$. This line width is typical for He I lines in other Type IIn events (e.g., SN 2005la, Pastorello et al. 2008). The total integrated flux in the early Keck spectrum is measured to be $\sim 5.5 \times$ $10^{-16} \mathrm{erg} \mathrm{s}^{-1} \mathrm{~cm}^{-2}$ by fitting a Lorentzian profile. It is possible that $\mathrm{Na} \mathrm{D}(\lambda \lambda 5890,5896)$ absorption contaminates this feature making it weaker. The $\mathrm{F}(\mathrm{H} \alpha) / \mathrm{F}\left(\mathrm{He}_{\mathrm{I}} 5876\right)$ flux ratio is $\sim 3$, probably reflecting the temperature/ionization conditions in the line-forming region. We do not detect the He II $4686 \AA$ line in any of our spectra.

\section{THE NATURE OF THE EMISSION LINES IN SN 2008am}

We conclude from the lack of broad SN features in the early-phase spectra of SN 2008am that the observed spectra are probably formed by CSM interaction, in which the SN ejecta collide with a dense CSM cloud surrounding the progenitor. In Section 4, we consider CSM interaction and other mechanisms to produce the light curve. Presuming CSM interaction plays some role, the collision should generate a double-shock pattern with the forward shock running into the CSM and the reverse shock propagating back into the ejecta. Between the shocks is a contact discontinuity, where a cool, dense shell (CDS) could form shortly after explosion. As the blast wave (the forward shock) runs through the dense CSM around SN 2008am, the temperature behind the shock is high enough to ionize both $\mathrm{H}$ and He. The emergent emission lines are thus expected to be due to radiative recombination. The photons can then further interact with the CSM in the early phases, resulting in the observed broadened emission lines.

The overall appearance of the intermediate-width Balmer emission lines on a nearly featureless, blue continuum makes SN 2008am similar to other Type IIn SNe, in particular the SLSN 2006gy (Smith et al. 2010). Here we use this similarity to address the possible line-forming mechanisms that may explain the observed spectral properties of SN 2008am.

\subsection{Possible Line-forming Processes}

Smith et al. (2010) delineated three phases for SN 2006gy. In the first phase, between 0 and 90 days after explosion (extending 20 days after maximum), when SN 2006gy was within a factor of two of peak light, $\mathrm{H} \alpha$ showed a nearly symmetric profile with no P Cygni features. Smith et al. associate this phase with conditions where there is a shock wave deep within a dense, opaque circumstellar shell, but the photosphere is in the outer, unshocked CSM. The $\mathrm{H} \alpha$ line is presumed to be excited by photoionization and to be intrinsically narrow, but broadened by multiple scattering on hot, free electrons (Fransson \& Chevalier 1989; Chugai 2001). In the second phase, 90-150 days after explosion (20-80 days after maximum light), the $\mathrm{H} \alpha$ line in SN 2006gy broadened somewhat, and developed a distinct, narrow P Cygni component with a velocity of $\sim 200 \mathrm{~km} \mathrm{~s}^{-1}$ and strong absorption in the blue wing extending to $\sim 4000 \mathrm{~km} \mathrm{~s}^{-1}$. The red wing is nearly constant during this phase and indicates a line of intermediate width of $\sim 1800 \mathrm{~km} \mathrm{~s}^{-1}$. Smith et al. attribute this phase to a condition where the photosphere has receded to beneath the forward shock so that radiation from the shocked matter can escape freely. Smith et al. (2010) proposed that the width of the red wing is determined by the Doppler shift of the expanding CDS that is presumed to form between the forward and reverse shocks in the collision of the SN ejecta with the dense CSM (Chugai 2001). The third phase in SN 2006gy is the very late phase, 150-240 days after explosion, when the $\mathrm{H} \alpha$ line becomes narrower. This is the phase when the CSM interaction is expected to decline in strength and the line-emitting region to become optically thin. SN 2008am and SN 2006gy had different rise times in their light curves, but their spectral evolutions show similarities if account is taken for the different timescales. 
Table 8

Gaussian, Lorentzian, and Exponential Fits to the Observed $\mathrm{H} \alpha$ and $\mathrm{H} \beta$ Line Profiles of SN 2008am

\begin{tabular}{|c|c|c|c|c|c|c|c|c|}
\hline Instrument $^{\mathrm{a}}$ & UT Date & Dayb $^{\mathrm{b}}$ & $\mathrm{FWHM}_{\mathrm{G}}^{\mathrm{c}}$ & $\mathrm{H} \alpha$ & $\sigma_{\exp }{ }^{c}$ & $\mathrm{FWHM}_{\mathrm{G}}{ }^{\mathrm{c}}$ & $\mathrm{H} \beta$ & $\sigma_{\exp }{ }^{c}$ \\
\hline & & & \multicolumn{3}{|c|}{ FWHM $_{\mathrm{L}}{ }^{\mathrm{c}}$} & \multicolumn{3}{|c|}{ FWHM $_{\mathrm{L}}{ }^{\mathrm{c}}$} \\
\hline HET-LRS & 2008 Jan 30.1 & +11 & 1562 & 1143 & 883 & 1956 & 1983 & 883 \\
\hline Keck-LRIS & 2008 Feb 12.0 & +22 & 1880 & 1441 & 1438 & 2179 & 2518 & 1283 \\
\hline Keck-LRIS $^{\mathrm{d}}$ & 2008 Feb 12.0 & +22 & 1073 & 755 & 551 & 1315 & 872 & 503 \\
\hline HET-LRS & 2008 Feb 18.3 & +27 & 1317 & 1003 & 796 & 1655 & 1657 & 796 \\
\hline HET-LRS & 2008 Feb 25.3 & +33 & 1351 & 1114 & 901 & 1042 & 1087 & 901 \\
\hline Keck-LRIS & 2009 Mar 31.0 & +352 & 1780 & 1142 & 918 & 280 & 190 & 148 \\
\hline
\end{tabular}

Notes.

a The HET-LRS and Keck-LRIS wavelength ranges are 4020-10200 ̊ and 3500-8600 A, respectively.

b All the values refer to the rest-frame days after maximum.

c The quantity FWHM $_{\mathrm{G}}$ corresponds to the fitted Gaussian, FWHM $\mathrm{L}$ to the fitted Lorentzian, and $\sigma_{\exp }$ to the fitted exponential profiles, all expressed in $\mathrm{km} \mathrm{s}^{-1}$.

d These estimates correspond to line profiles not corrected for host extinction.

We first examine the possibility that the intermediate-width lines in the early phase of SN 2008am obtain their wings from multiple electron scattering, a mechanism favored by Smith et al. (2010) for SN 2006gy. Our first four spectra of SN 2008am were obtained when the SN was within a factor of two of maximum light, in keeping with Phase 1 of SN 2006gy by Smith et al. Our spectra can be fit well with a single Lorentzian profile (see below) consistent with multiple electron scattering, and they show no sign of broad features nor P Cygni lines of any width, similar to Phase 1. For these reasons, we believe a plausible case can be made that, despite the different timescales, SN 2008am could be a close cousin to SN 2006gy, and that our early-phase spectra are formed in the phase when the photosphere was in a dense CSM shell, but beyond the forward shock. If this is the case, SN 2008am could very well have then proceeded to Phase 2 defined by Smith et al. (2010), but we simply failed to obtain any spectra in this phase, more than 2 mag below maximum light. At very late phases, the two objects displayed substantially narrower $\mathrm{H} \alpha$ profiles and are again quite similar. Further support for this model may come from the fact that in the early phase both $\mathrm{H} \alpha$ and $\mathrm{H} \beta$ showed peaks redshifted by $\sim 100 \mathrm{~km} \mathrm{~s}^{-1}$ from their rest-frame wavelength. The same effect was observed for SN 2006gy by Smith et al. (2010) and it is also predicted by the electron scattering model of Chugai (2001). Chugai (2001) assumed a velocity profile in which the velocity decreased with radius as might be caused by radiative acceleration. Fransson \& Chevalier (1989) assumed a homologous velocity profile in their multiple scattering models and found profiles with enhanced red wings and a small blue shift of the peak. The details of the line profiles might thus contain information on the velocity profiles in the scattering regions of SN 2008am and related events, but we have not explored this in any depth.

The second possibility is that the intermediate-width lines arise from the post-shock motion of the shocked CSM in a CDS when the photosphere is interior to the forward shock (Fransson 1984; Chugai et al. 2004; Dessart et al. 2009). This would correspond to our early spectra being already in the Phase 2 of Smith et al. (2010). The principal argument against this interpretation is that we do not see any of the narrow or broad absorption manifested by SN 2006gy when it was in this phase, as interpreted by Smith et al. (2010). It could be that the structure of the CSM around SN 2008am is such that the matter interior to and beyond the shock is not sufficiently optically thick to create appreciable absorption. Another possibility is that these absorption features might have been observed had we had better $\mathrm{S} / \mathrm{N}$ and/or better spectral resolution. We thus cannot rule out this possibility, but find it somewhat less likely than the broadening by multiple electron scattering.

Another mechanism that has been proposed to account for intermediate-width lines is the inward propagation of shocks into dense clumps of matter that have been engulfed by the SN shock (Chugai \& Danziger 1994), rather than a single postshock shell. In this picture, the SN shock sweeps past clumps in the progenitor wind, but the resulting high pressure of the shocked low-density CSM drives a shock into the dense clumps. This may broaden the lines via Doppler motion, and for appropriate choices of cloud sizes, densities, and other parameters one can produce $\mathrm{H} \alpha$ lines of suitable width and intensity. The problem with this model in the current context is that it is designed to have dense clumps separated by less dense, optically thin material. The latter should allow the SN ejecta to be observed directly. Since we see no sign of high-velocity SN features, we find the configuration with the enveloping CDS, as attributed to SN 2006gy by Smith et al. (2010), to be preferable to the clumpy model, where the dense clumps have a rather small filling factor.

\subsection{Line Profile Fitting}

In the context of the CSM interaction picture, we considered three different models to account for the line profiles of the earlyphase spectra of SN 2008am: (1) thermal Doppler broadening producing Gaussian line shapes, (2) single Thompson scattering on free electrons giving exponential profiles (Laor 2006), and (3) multiple scattering on hot free electrons resulting in Lorentzian profiles (Chugai 2001; Smith et al. 2010). The first phenomenon is common in stellar atmospheres. The second one is proposed for the broad-line region in active galactic nuclei (AGNs)/quasistellar objects. The third one might pertain to the dense CSM environment around Type IIn SNe.

We fitted Gaussian, Lorentzian, and exponential profiles to the observed features. The results are listed in Table 8 and plotted in Figure 12. The Gaussian fits were inferior compared to the other two models in terms of conforming to profile shapes. Gaussian models resulted in FWHM $=1500 \pm 300 \mathrm{~km} \mathrm{~s}^{-1}$ for $\mathrm{H} \alpha$ and $1600 \pm 500 \mathrm{~km} \mathrm{~s}^{-1}$ for $\mathrm{H} \beta$. If interpreted as a simple thermal Doppler broadening, the corresponding temperature would be $\sim 10^{8} \mathrm{~K}$, which is obviously too high. On the other hand, if the line width is attributed to bulk kinematic motion, these velocities are too low to be directly related to the expected velocities of SN ejecta. 

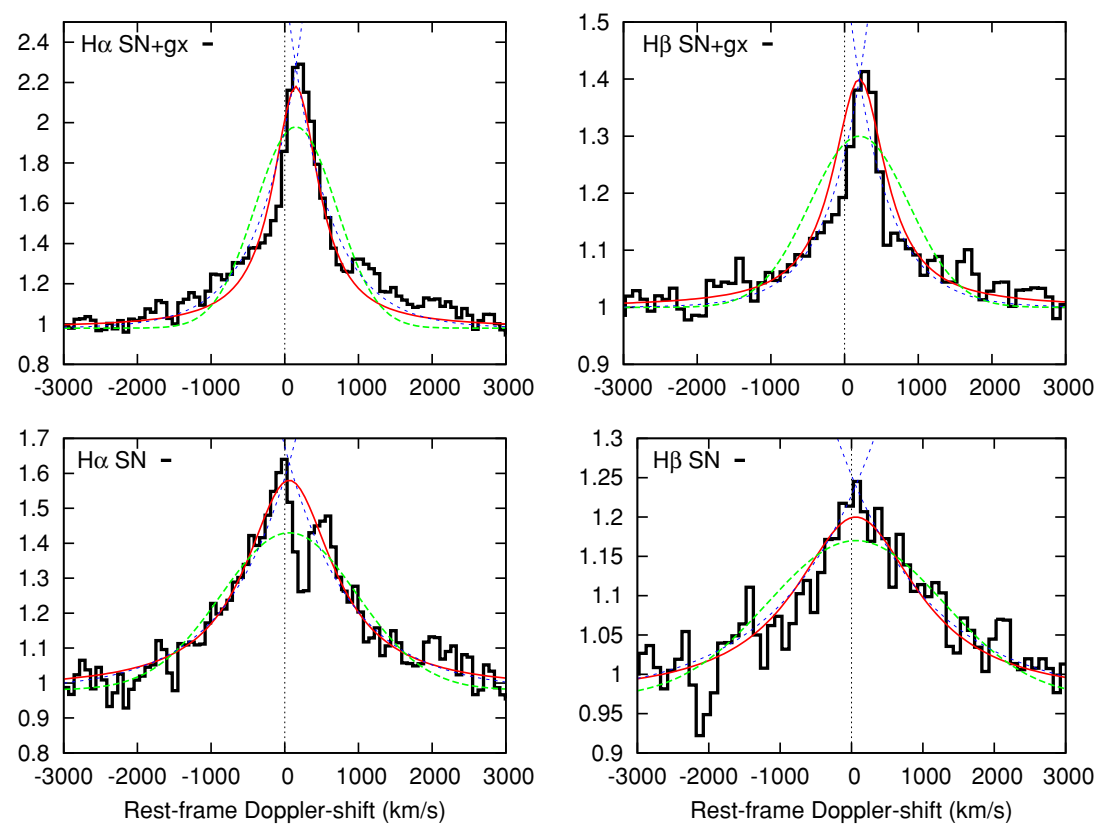

Figure 12. Gaussian (dashed green curve), Lorentzian (solid red curve), and exponential (dotted blue curve) fits to the $\mathrm{H} \alpha$ (upper and lower left panels) and $\mathrm{H} \beta$ (upper and lower right panels) line profiles from the Keck spectrum +22 rest-frame days after maximum. In each case, the upper panel shows the fits to the line profile not corrected for host contribution and the lower panel shows the fits to the host subtracted line profile. Lorentzian profiles provide the best overall fit to the observed line profiles (see the text).

(A color version of this figure is available in the online journal.)

The exponential fits are motivated by the possibility that the line-forming region might be a photoionized, but less dense, optically thin medium, similar to the environment of AGNs. In this case, the observed line profiles are due to Thompson scattering on hot free electrons. If the medium is less dense, hence optically thin to electron scattering, single scattering is an adequate description. Laor (2006) investigated such a region and derived the emergent line shape to $b e \sim \exp (-\Delta v / \sigma)$, where $\Delta v$ is the Doppler shift from the line center and $\sigma$ is the velocity. The electron-scattering optical depth can be expressed as $\tau_{e}=\exp \left(-\sigma /\left(1.1 \sigma_{e}\right)\right)^{2.222}$ where $\sigma_{e} \sim\left(k T_{e} / m_{e}\right)$ is the velocity dispersion of free electrons. The fits to the observed line profiles resulted in $\sigma=800 \pm 100 \mathrm{~km} \mathrm{~s}^{-1}$, which gives $\tau_{e}$ $\sim 0.03$ if the electron temperature $T_{e}$ is assumed to be $11,000 \mathrm{~K}$, close to the continuum effective temperature of SN 2008am. The resulting optical depth is much below unity, verifying the general assumption of this model; however, it should be noted that higher optical depths produce narrower lines in this model. Since we observe intermediate-width lines earlier and a narrower line later, the prediction from the Laor model would be the strong increase of electron optical depth, the opposite of what is expected in an expanding, diluting SN environment.

The fitting of Lorentzians produced the best fit to all line profiles. The resulting FWHMs are $\sim 1000 \pm 300 \mathrm{~km} \mathrm{~s}^{-1}$ for $\mathrm{H} \alpha$ and $\sim 1700 \pm 500 \mathrm{~km} \mathrm{~s}^{-1}$ for $\mathrm{H} \beta$. The narrower $\mathrm{H} \alpha$ profile may be the consequence of host contribution to the HET spectra, which is certainly stronger in $\mathrm{H} \alpha$. Indeed, the FWHM of the host-subtracted early-phase Keck $\mathrm{H} \alpha$ profile is much closer to the average FWHM of the $\mathrm{H} \beta$ line. Since the line-forming medium is assumed to be optically thick in this model, the line widths from the Lorentzian fitting cannot be simply related to the physical conditions characterizing the whole line-forming region. A higher density of free electrons should produce wider profiles and prevent the direct escape of the original narrowwidth line. As a consequence, as the line-forming medium expands, the line broadening should decrease.
If multiple scattering is the correct interpretation, then the width of the lines we measure in the early-phase spectra of SN 2008am cannot be attributed to bulk kinematic motion. The width of the lines gives an upper limit on the motion of the lineforming region but yields no constraint on the velocity of deeper, optically thick regions. The profiles may give information on the velocity distribution in the scattering region, since the velocity profile can affect the resulting scattering-line profile if the bulk velocity exceeds the electron thermal velocity (Fransson \& Chevalier 1989; Chugai 2001). For multiple scattering, the line wings are primarily a measure of the electron scattering optical depth of the line-emitting region. Following Smith et al. (2010), we estimate the Thompson scattering optical depth from the formula $U=\left(1-e^{-\tau_{T}}\right) / \tau_{T}$, where $U$ is the ratio of the narrow, unscattered $\mathrm{H} \alpha$ line flux to the total $\mathrm{H} \alpha$ luminosity. Since we were unable to fully resolve the narrow component in the +358 day Keck spectrum, we use a conservative estimate of $U \leqslant 0.5$. This results in a lower limit of $\tau_{T} \geqslant 2$ for SN 2008am (Smith et al. obtained $\tau_{T} \sim 15$ for SN 2006gy), consistent with an optically thick scattering medium.

\section{MODELS FOR SN 2008am}

Figure 13 shows the ROTSE unfiltered light curve of SN 2008am compared to some other SLSNe and confirms that SN 2008am is one of the brightest SNe ever observed. Figure 13 also shows that the late photospheric evolution of SN 2008am (after $\sim 120$ days) in the $R$ band is very similar to that of SN 2006tf and slower compared to all the other SLSNe except SN 2003ma (Rest et al. 2009). In the following discussion, we use the derived rise time and ROTSE light curve of SN 2008am (Section 2.2; Figure 3) to obtain estimates of the mass of involved ejecta+CSM and to discuss possible power sources for this exceptional stellar explosion. To do so, we fit light curve models that account for the diffusion of radiated energy deposited from a variety of power sources. The fitting 


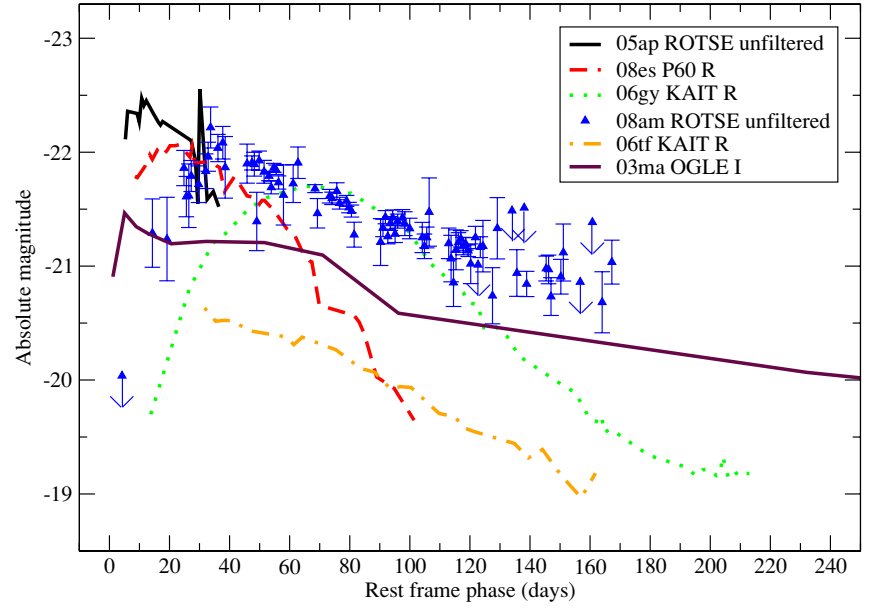

Figure 13. Comparison of the rest-frame light curve of SN 2008am with those of other luminous supernovae: SN 2003ma (Rest et al. 2009), SN 2005ap (Quimby et al. 2007a), SN 2006gy (Smith et al. 2007), SN 2006tf (Smith et al. 2008), and SN 2008es (Gezari et al. 2009).

(A color version of this figure is available in the online journal.)

in each case is performed by Monte Carlo $\chi^{2}$ minimization as described in Chatzopoulos et al. (2009). We also compare to our derived SED light curves, as appropriate.

\subsection{Radioactive Decay Diffusion Models}

Although there is no evidence for a classic SN photosphere, the light curve of SN 2008am (Figure 3) is reminiscent of SNe powered by radioactive decay. The first model that we consider for SN 2008am is thus one of radioactive decay diffusion that was developed by Arnett $(1980,1982)$ and generalized by Valenti et al. (2008; see also supplementary information in Soderberg et al. 2008). In this model, the power source of the SN luminosity is the radioactive decay of nickel and cobalt, the energy of which diffuses out from the expanding envelope. This model was developed in the context of Type Ia $\mathrm{SNe}$ and is appropriate in the absence of an $\mathrm{H}$ recombination phase with a constant opacity in the photospheric phase (the effects of $\mathrm{H}$ recombination were considered by Arnett \& Fu 1989 and Chatzopoulos et al. 2009). The light curve is given by the following formula (Valenti et al. 2008; Chatzopoulos et al. 2009):

$$
\begin{aligned}
L(t)= & M_{\mathrm{Ni}} e^{-x^{2}}\left[\left(\epsilon_{\mathrm{Ni}}-\epsilon_{\mathrm{Co}}\right) \int_{0}^{x} 2 z e^{z^{2}-2 z y} d z+\epsilon_{\mathrm{Co}}\right. \\
& \left.\times \int_{0}^{x} 2 z e^{z^{2}-2 y z+2 z s} d z\right]\left(1-e^{-A t^{-2}}\right),
\end{aligned}
$$

where $x=t / t_{m}, t_{m}$ is the effective diffusion time which is generally close to the rise time to maximum, $y=t_{m} / 2 t_{\mathrm{Ni}}$ with $t_{\mathrm{Ni}}=8.8$ days, $s=t_{m}\left(t_{\mathrm{Co}}-t_{\mathrm{Ni}}\right) / 2 t_{\mathrm{Co}} t_{\mathrm{Ni}}$ with $t_{\mathrm{Co}}=111.3$ days, $M_{\mathrm{Ni}}$ is the initial nickel mass, and $\epsilon_{\mathrm{Ni}}=3.9 \times 10^{10} \mathrm{erg} \mathrm{s}^{-1} \mathrm{~g}^{-1}$ and $\epsilon_{\mathrm{Co}}=6.8 \times 10^{9} \mathrm{erg} \mathrm{s}^{-1} \mathrm{~g}^{-1}$ are the energy generation rates due to $\mathrm{Ni}$ and $\mathrm{Co}$ decay. The factor $\left(1-e^{-A t^{-2}}\right)$ accounts for the gamma-ray leakage, where large $A$ means that practically all gamma rays are trapped. The gamma-ray optical depth of the ejecta is taken to be $\tau_{\gamma}=\kappa_{\gamma} \rho R=A t^{-2}$, assuming spherical uniform density ejecta with radius $R=v t$ and the $\mathrm{Ni} / \mathrm{Co}$ confined in the center. This yields $A=\left(3 \kappa_{\gamma} M_{\mathrm{ej}}\right) /\left(4 \pi v^{2}\right)$ which is controlled by the gamma-ray opacity, $\kappa_{\gamma}$. The $t^{-2}$ scaling follows from homologous expansion, which is one of the basic assumptions of the simple analytic models that we adopt here. Thus, the main parameters of this model are the nickel mass, $M_{\mathrm{Ni}}$, and the effective diffusion time $t_{m}$, which corresponds to an ejecta mass as given by the following equation for a constant density envelope:

$$
M_{\mathrm{ej}}(z)=\frac{3}{10} \frac{\beta c}{\kappa} v \frac{t_{m, \mathrm{ob}}^{2}}{(1+z)^{2}},
$$

where $\beta$ is an integration constant equal to about 13.8 (Arnett 1982; Valenti et al. 2008), $\kappa$ is the mean optical opacity, $v$ the mean photospheric expansion velocity, $t_{m, \mathrm{ob}}$ the observed rise time, and $z$ the redshift of the SN. As described in Section 3.2, we have concluded that the observed emission lines are dominated by multiple electron scattering so that their width yields no direct information on the bulk kinematic expansion velocity. In the following, we will scale our results to a characteristic velocity of $v=1000 \mathrm{~km} \mathrm{~s}^{-1}$ that is consistent with the limit set by line widths and might be compatible with velocities expected for shocks traversing dense circumstellar shells.

We provide a characteristic fit of the radioactive decay diffusion model to the ROTSE light curve of SN 2008am in order to estimate model parameters for this SN and to determine the applicability of this model. For the ROTSE light curve of SN 2008am the explosion date is held fixed, established in Section 2.2. Thus, we are left with three fitting parameters: $M_{\mathrm{Ni}}$, $t_{m}$, and $A$. The gamma-ray leakage in this model is very small over the range of our observations. A decent fit is obtained for $M_{\mathrm{Ni}}=19 M_{\odot}$ and $t_{m}=41$ days. The 41 day effective diffusion time corresponds to an ejecta mass $M_{\mathrm{ej}}=0.2 M_{\odot}$, using Equation (2) with the fiducial values $\kappa=0.4 \mathrm{~cm}^{2} \mathrm{~g}^{-1}$, appropriate for Thompson scattering in a pure $\mathrm{H}$ plasma, and $v=1000 \mathrm{~km} \mathrm{~s}^{-1}$. If we use $v=2000 \mathrm{~km} \mathrm{~s}^{-1}$ instead, which would be above the upper limit for the velocity implied from the observed FWHM of the emission lines in the early spectra of SN 2008am, and adopt a lower value for the optical opacity, $\kappa=0.05 \mathrm{~cm}^{2} \mathrm{~g}^{-1}$, characteristic of metal-rich ejecta, the ejecta mass can be scaled up to $3.2 M_{\odot}$. Even for this low opacity, the velocity would have to be $v \sim 12,000 \mathrm{~km} \mathrm{~s}^{-1}$ for the diffusion mass to exceed the required nickel mass, and we have no rationale to adopt such a high velocity.

The left panel of Figure 14 shows the radioactive decay diffusion model (red solid curve) fitted to the ROTSE light curve of SN 2008am (blue filled squares). The radioactive decay rate of cobalt for $M_{\mathrm{Ni}}=19 M_{\odot}$ is shown as the red dashed curve in the same plot for comparison. The light blue data points correspond to the pseudo-bolometric light curve of SN 2008am derived by the SED fits. As we have shown above, the value of $M_{\mathrm{ej}}$ would be greater for lower $\kappa$ and higher $v$, but it never becomes equal to or higher than the nickel mass for reasonable choices of these parameters given the short rise time of SN 2008am. This makes the radioactive decay diffusion model unphysical for this event. Although a large amount of radioactive nickel has been suggested for some SLSNe as a product of pair-instability (for example, $22 M_{\odot}$ for SN 2006gy; Smith et al. 2007 and $4.5 M_{\odot}$ for SN 2006tf; Smith et al. 2008), the inconsistency between the total ejected mass and the nickel mass in SN 2008am is quite remarkable. Integrating under the solid red curve of the left panel of Figure 14 yields a total radiated energy output $E_{\text {rad }} \simeq 2.1 \times 10^{51} \mathrm{erg}$. On the other hand, the kinetic energy of the explosion in this model, $E_{\mathrm{KE}}=(1 / 2) M_{\mathrm{ej}} v_{\text {mean }}^{2}$ is $0.6 \times 10^{48}$ erg where $v_{\text {mean }}=\sqrt{3 / 5} v$ assuming $v=1000 \mathrm{~km} \mathrm{~s}^{-1}$ and for $\kappa=0.4 \mathrm{~cm}^{2} \mathrm{~g}^{-1}$. Even within 

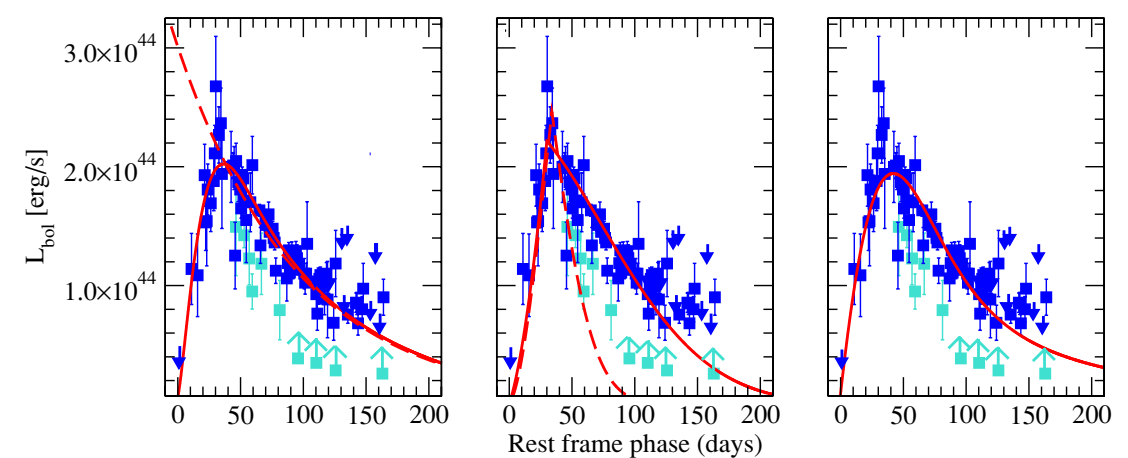

Figure 14. Characteristic fits of various supernova light curve models to the ROTSE light curve of SN 2008am (filled blue squares; downward arrows represent upper limits). See the text (Section 4) for details on the model fitting and the derived best-fitting parameters of each model. The light blue data points correspond to the pseudo-bolometric light curve of SN 2008am derived by the SED fits for comparison. Left panel: fit of a radioactive decay model (solid red curve) with $M_{\mathrm{Ni}}=$ $19 M_{\odot}$ and $t_{d}=41$ days. The radioactive energy decay rate for the same amount of radioactive nickel is shown for reference (dashed red curve). Middle panel: fit of a shell-shock diffusion model (optically thick CSM, or hybrid model) for $t_{d}=t_{\max }=34$ days (dashed red curve) and for $t_{d}>t_{\max }$ (solid red curve). The $t_{d}=t_{\max }$ model cannot fit the light curve of SN 2008am. Right panel: fit of a magnetar spin-down model (solid red curve).

(A color version of this figure is available in the online journal.)

the uncertainties of these estimates, the kinetic and total radiated energy are also found to be inconsistent. Radioactive decay may contribute to the output energy, but the most significant contribution must come from other mechanisms. We discuss other models in the following sections.

\subsection{Shell-shock Diffusion Model}

Here we consider a shell-shock diffusion model, similar to that suggested by Smith \& McCray (2007) for SN 2006gy. In this type of model, the energy that powers the SN light curve is produced by the diffusion of shock-generated energy through an optically thick CSM shell of large initial radius. Smith \& McCray (2007) considered the diffusion of shock-generated energy in a homologously expanding CSM shell in the case where the shock energy input is instantaneous and at the time of maximum light. This type of model does not account for the observed rise of the light curve. Smith \& McCray (2007) adopted an $L \propto r^{2}$ rise for their model of SN 2006gy based on the early portion of the diffusion models of Arnett (1982). They did not self-consistently consider the input necessary to drive such a rise. They found the diffusion time on the decline to be about the observed rise time for SN 2006gy, but we consider that a coincidence, since such a model fails to account for SN 2008am, as we show below. In order to account for the rising part of the light curve of SN 2008am, we consider a forward shock that propagates through the CSM envelope and deposits energy for a time $t_{\mathrm{sh}}=t_{\max }$ and then shuts off. The effect of the reverse shock will be considered below. This model is somewhat similar to the "top hat" magnetar-input model that is considered in Kasen \& Bildsten (2010) with the exception that we solve for the general case of large initial radius. The luminosity deposition function in our model has the form $L_{\mathrm{sh}}(t)=E_{\mathrm{sh}} / t_{\mathrm{sh}}$ for $t<t_{\mathrm{sh}}$ and $L_{\mathrm{sh}}=0$ otherwise, where $E_{\mathrm{sh}}$ is the total kinetic energy deposited by the shock in the CSM shell. This model formally assumes that the luminosity $E_{\mathrm{sh}} / t_{\mathrm{sh}}$ is deposited in the center of homologously expanding matter. While not totally selfconsistent, this model captures the essence of the shell-shock model on both the rise and decline. Using this energy deposition function and the first law of thermodynamics coupled with the diffusion approximation (as was originally done by Arnett 1980, but for a radioactive decay input), it can be shown that the light curve is given by the following expression:

$L(t)= \begin{cases}\frac{E_{\mathrm{sh}}}{t_{\mathrm{sh}}}\left[1-e^{-\left(t^{2} / 2 t_{d}^{2}+R_{0} t / v t_{d}^{2}\right)}\right], & t<t_{\mathrm{sh}}, \\ \frac{E_{\mathrm{sh}}}{t_{\mathrm{sh}}} e^{-\left(t^{2} / 2 t_{d}^{2}+R_{0} t / v t_{d}^{2}\right)}\left[e^{\left(t_{\mathrm{sh}}^{2} / 2 t_{d}^{2}+R_{0} t_{\mathrm{sh}} / v t_{d}^{2}\right)}-1\right], & t>t_{\mathrm{sh}},\end{cases}$

where $t_{d}$ is the diffusion timescale, $v$ is the characteristic bulk velocity of the CSM shell, and $R_{0}$ is the initial radius of the optically thick CSM shell around SN 2008am. We note that in the case where $R_{0}$ is small, the result for small initial radius is recovered (Kasen \& Bildsten 2010).

To evaluate this model, the second part of Equation (3) $\left(t>t_{\mathrm{sh}}\right)$ is fitted to the ROTSE light curve decline of SN 2008am in order to determine $E_{\mathrm{sh}}, R_{0}$, and $t_{d}$. Then using the best-fitting parameters, we plot the expected rise to maximum for the event within this class of model. We first consider the case for which the diffusion time on the decline is forced to be equal to the input time, which is also the rise time to maximum $\left(t_{\max }=\right.$ 34 days). The best fit to the data in this case, is obtained for $E_{\mathrm{sh}}=1.6 \times 10^{51} \mathrm{erg}$ and $R_{0}=3.0 \times 10^{13} \mathrm{~cm}$ if $R_{0}$ is constrained to be larger than the smallest possible progenitor radius $\left(10^{11} \mathrm{~cm}\right.$ for Wolf-Rayet stars). Forcing $t_{d}$ on the decline to be equal to the rise time of 34 days means the light curve falls below the indicated lower limits. The result of this failed "fit" is shown as the red dashed curve in the middle panel of Figure 14. We next consider the general case where $t_{d}>t_{\mathrm{sh}}$, and we also fit $t_{d}$ as a free parameter. The best fit is obtained for $E_{\mathrm{sh}}=5.5 \times 10^{51} \mathrm{erg}, R_{0}=1.0 \times 10^{14} \mathrm{~cm}$, and $t_{d}=87$ days. The result of this fit is shown as the red solid curve in the middle panel of Figure 14. The 87 day diffusion time corresponds to a CSM shell having a mass $M_{\text {shell }}=1.0 M_{\odot}$ (using Equation (2), again for $v=1000 \mathrm{~km} \mathrm{~s}^{-1}$ and $\kappa=0.4 \mathrm{~cm}^{2} \mathrm{~g}^{-1}$ ). For the extreme values of $v=2000 \mathrm{~km} \mathrm{~s}^{-1}$ and $\kappa=0.05 \mathrm{~cm}^{2} \mathrm{~g}^{-1}$ the shell mass is $16 M_{\odot}$. The radius of the shell at maximum light adopting a constant expansion velocity equal to $1000 \mathrm{~km} \mathrm{~s}^{-1}$ is $R_{\max }=3.7 \times 10^{14} \mathrm{~cm}$. These results are consistent with a relatively large optically thick CSM shell around SN 2008am. The total radiated energy within the context of this model is equal to $10^{51} \mathrm{erg}$. Note that the rise in this simple "top hat" model is concave upward rather than convex.

We conclude that the derived parameters for the CSM shell of SN 2008am based on a shell-shock diffusion model show that 
it may be less massive and smaller than the ones determined for SN 2006gy $\left(M_{\text {shell }}=10 M_{\odot}, R_{\text {shell }}=2.4 \times 10^{15} \mathrm{~cm}\right.$; Smith $\&$ McCray 2007) and for SN 2006tf $\left(M_{\text {shell }}=18 M_{\odot}, R_{\text {shell }}=\right.$ $2.7 \times 10^{15} \mathrm{~cm}$, Smith et al. 2008 using the same type of model). It should be noted that the estimates for the properties of the shell of SN 2006tf are very uncertain due to the lack of data during the rising part of the light curve and thus the lack of an accurate explosion date. The larger mass derived for SN 2006gy is mainly determined by the larger rise time of 70 days versus 34 days for SN 2008am and by the larger velocity of $4000 \mathrm{~km} \mathrm{~s}^{-1}$ versus the fiducial $1000 \mathrm{~km} \mathrm{~s}^{-1}$ that we have adopted for SN 2008am.

An estimate of the optical depth of the CSM shell under the assumption that the derived shell radius is significantly larger than the radius of the progenitor and for constant density profile is given by

$$
\tau_{\text {shell }}=\frac{3 \kappa M_{\text {shell }}}{4 \pi R_{\text {shell }}^{2}} .
$$

Assuming that electron scattering in a hydrogen plasma is the main source of opacity $\left(\kappa=0.4 \mathrm{~cm}^{2} \mathrm{~g}^{-1}\right), v=1000 \mathrm{~km} \mathrm{~s}^{-1}$ (and taking for the CSM shell the derived parameters $M_{\text {shell }}=$ $1.0 M_{\odot}$ and $R_{\text {shell }}=R_{\max }=3.7 \times 10^{14} \mathrm{~cm}$ ) an estimate for the optical depth of the shell around SN 2008am at maximum light is $\tau_{\max } \sim 1390$ while for SN 2006tf it is $\tau_{\max } \sim 480$ (Smith et al. 2008) and for SN 2006gy it is $\tau_{\max } \sim 330$ (Smith et al. 2007). Thus, although less massive, the CSM shell of SN 2008am is very optically thick due to its small radius compared to the shells of those other SLSNe, which results in higher density. The values of the optical depth will vary for different choices of optical opacity. For the derived optical depth, we can estimate when the shell will become optically thin. Since $\tau \propto R^{-2}, R_{2}=R_{\max } \sqrt{\tau_{\max } / \tau_{2}}$ for a constant expansion velocity, where $R_{2}=R_{\max }+v \Delta t$. Combining those two equations yields $\Delta t=R_{\max }\left(\sqrt{\tau_{\max } / \tau_{2}}-1\right) / v$. Thus, we can estimate how long will it take for the CSM to become optically thin $\left(\tau_{2}=1\right)$ for $\tau_{\max }=3600$ and $R_{\max }=3.7 \times 10^{14} \mathrm{~cm}$. This yields $\Delta t \simeq 4.3$ yr for $v=1000 \mathrm{~km} \mathrm{~s}^{-1}$.

An estimate for the mass-loss rate is given by $\dot{M}=$ $M_{\text {shell }} v_{w} /\left(t_{\max } v\right)$. For SN 2008am $\left(M_{\text {shell }}=1.0 M_{\odot}\right)$, we find $\dot{M}=0.9 \times 10^{-2} v_{w} M_{\odot} \mathrm{yr}^{-1}$ for $v=1000 \mathrm{~km} \mathrm{~s}^{-1}$ and with $v_{w}$ in units of $\mathrm{km} \mathrm{s}^{-1}$. For the range of wind velocities $10 \mathrm{~km} \mathrm{~s}^{-1}$ $<v_{w}<1000 \mathrm{~km} \mathrm{~s}^{-1}$ the inferred mass-loss rates range from 0.1 up to $10 M_{\odot} \mathrm{yr}^{-1}$. These extraordinary mass-loss rate estimates imply a very massive luminous blue variable (LBV)-type progenitor for SN 2008am, as was suggested for SN 2006gy (Smith et al. 2008). We note again that the choice of $1000 \mathrm{~km} \mathrm{~s}^{-1}$ as the expansion velocity of the circumstellar shell, which was constrained by fitting Lorentzian profiles to the Balmer emission lines in the early spectra of SN 2008am (see Section 3.2), is just for illustration purposes.

We also investigated the possibility that SN 2008am is powered by the reverse shock that we expect to have formed due to an ejecta-CSM interaction. Chevalier \& Fransson (2003) estimate the density of the swept up matter behind the reverse shock to be

$$
\rho_{\mathrm{rev}}=\frac{(-n-4)(-n-3)}{2} \rho_{\mathrm{CSM}},
$$

where $n$ is a constant that describes the density profile of the ejecta $\left(\rho_{e j} \propto R^{n}\right)$. The density behind the reverse shock is higher than that behind the forward shock for $n \leqslant-7$. The luminosity from the reverse shock scales as $L_{\text {rev }} \propto t^{-3 /(-n-2)}$. The reverse shock may be adiabatic or radiative depending on the optical depth of the shell. We fit a power law of the form $L=A t^{p}$ to the decline of the observed ROTSE light curve in order to get an estimate of $n$ and determine whether this model can account for SN 2008am. This procedure effectively assumes that the reverse shock dominates the forward shock and that the diffusion time in the reverse-shocked gas is small. The best-fit power-law model gives $p=-1.1 \pm 0.3$ which corresponds to $n=-4.7 \pm 0.1$. Since $n>-7$ we conclude that the density behind the reverse shock may be smaller than that behind the forward shock and hence that the luminosity from the reverse shock may be lower than that of the forward shock.

\subsection{Optically Thin CSM-Ejecta Interaction}

A related model for the luminosity source for SN 2006gy (Ofek et al. 2007), SN 2002ic (Hamuy et al. 2003) and SN 2005gj (Aldering et al. 2006) is that of the interaction between the ejecta and the CSM in the case where the latter is dense, but optically thin. In such a model, a radiative shock forms due to the collision between the ejecta and the CSM (Chevalier $\&$ Fransson 1994). We consider the luminosity produced by the forward shock. The luminosity in this case given by (Ofek et al. 2007)

$$
L=2 \pi \rho_{\mathrm{CSM}} R^{2} v_{\mathrm{sh}}^{3},
$$

where $\rho_{\mathrm{CSM}}$ is the local CSM density, $R=v_{\mathrm{sh}} t$ is the radius of the shock at time $t$ and $v_{\text {sh }}$ the velocity of the shock. Assuming that the shock enters the optically thin CSM envelope at a radius $R_{0}$ and that the density profile follows a power law of the form $\rho=\rho_{0}\left(R / R_{0}\right)^{m}$, where $R=R_{0}+v_{\mathrm{sh}} t$ and $m$ is the slope of the density profile (for a constant velocity wind $m=-2$ ), we can rewrite Equation (6) as

$$
L=2 \pi \rho_{0} R^{2} v_{\mathrm{sh}}^{3}\left[\left(R_{0}+v_{\mathrm{sh}} t\right) / R_{0}\right]^{m} .
$$

In our analysis, we assume that the shock velocity is constant for simplicity. More accurate solutions that take the change of the shock velocity into account can be found in Chevalier (1982) and Chevalier \& Fransson (2003). If the CSM around the progenitor star is everywhere optically thin, $R_{0}$ represents the radius of the progenitor star.

An estimate for an upper limit of the mass-loss rate of the progenitor of SN 2008am in the context of an optically thin CSM comes from the X-ray flux upper limit measurement (see Section 2.2). According to Immler \& Kuntz (2005), the X-ray luminosity from thermal Bremsstrahlung is given by

$$
L_{X}=\frac{4}{(\pi m)^{2}} \Lambda(T)\left(\frac{\dot{M}}{v_{w}}\right)^{2}\left(v_{\mathrm{sh}} t\right)^{-1},
$$

where $L_{X}$ is the X-ray luminosity, $m_{p}$ the mean mass per particle (we adopt $m_{p}=2.1 \times 10^{-24} \mathrm{~g}$ for an $\mathrm{H}+\mathrm{He}$ plasma), and $\Lambda(T)$ the cooling function of a plasma that has temperature $T$. As in Immler \& Kuntz (2005), we adopt $\Lambda(T)=3 \times 10^{-23}$ erg $\mathrm{cm}^{-3} \mathrm{~s}^{-1}$ for $T=10^{7} \mathrm{~K}$ which is characteristic of the post-shock temperature. For the available XRT upper limit of $L_{X}=10^{43} \mathrm{erg} \mathrm{s}^{-1}$ corresponding to $\sim 50$ rest-frame days after explosion and 16 days after maximum, we obtain an upper limit of $\dot{M}=0.07\left(v_{w} / 1000 \mathrm{~km} \mathrm{~s}^{-1}\right) M_{\odot} \mathrm{yr}^{-1}$. It should be noted that this model assumes a constant wind-density parameter $w=\dot{M} / v_{w}$.

Most of the radiation that is produced in models of the radiative forward shock is emitted at ultraviolet and X-ray wavelengths. We assume in the context of this model that the 
ROTSE light curve is a good proxy to the true bolometric light curve of SN 2008am as would be the case if a substantial fraction of UV/X-ray luminosity is absorbed and re-radiated by the shell that forms behind the forward shock. Then, the best fit of Equation (7) to the ROTSE light curve of SN 2008am would provide us with estimates of $\rho_{0}, R_{0}$, and $m$.

The optical depth of the CSM is

$$
\tau_{\text {thin }}=\kappa \int_{R_{0}}^{\infty} \rho d R=-\frac{\kappa \rho_{0} R_{0}}{m+1},
$$

where $m<-1$. In our fitting process, we demand that this optical depth beyond $R_{0}$ is less than one for $\kappa=0.4 \mathrm{~cm}^{2} \mathrm{~g}^{-1}$. Thus, we choose the best-fit model for which this condition is met in order to be self-consistent with the initial assumption of an optically thin CSM. We were unable to determine a satisfactory fit of this simple optically thin model to the ROTSE light curve of SN 2008am that was self-consistent, in terms of the optical depth. For all the best-fit models the optical depth was well above unity, and for models with $\tau_{\text {thin }}<1$ the derived values for the density and the radius were unphysical.

A purely optically thin model not only fails to account for the light curve of SN $2008 \mathrm{am}$ but also for the observed spectroscopic characteristics. Chevalier (1982) and Chevalier \& Fransson (2003) predict that the bulk of the luminosity from optically thin forward shock emission is in the UV and X-ray region of the spectrum. It seems unlikely that the optically thin case would reproduce the spectra and spectral evolution of SN 2008am. In addition, this model being optically thin, it is incompatible with the failure to see SN photospheric features. We conclude that an optically thin ejecta-CSM interaction model alone does not provide a satisfactory explanation for SN 2008am.

\subsection{Hybrid CSM Interaction Model}

Next, we discuss a hybrid model in which the CSM comprises both an optically thick and an optically thin region. This is somewhat similar to the model proposed by Chugai \& Danziger (1994) in which the CSM contains two components: an optically thin rarefied wind and optically thick clumps or an optically thick disk around the progenitor. In this model, the light curve is described by the sum of Equations (3) and (7). We assume the whole structure follows a single power-law density profile $\rho \propto R^{m}$ so that the optical depth at a given point is

$$
\tau=\frac{-\kappa \rho_{0} R}{m+1}\left(\frac{R}{R_{0}}\right)^{m} .
$$

We take the point $\tau=1$ at radius $R=R_{1}$ to represent the boundary between the optically thick and optically thin regions. In this case, $R_{0}$ corresponds to the inner initial radius of the optically thick part of the CSM shell, which is also the radius of the progenitor star, where the density is $\rho_{0}$. The radius of unity optical depth is $R_{1}=\left[-R_{0}^{m}(m+1) /\left(\kappa \rho_{0}\right)\right]^{1 /(m+1)}$, and we demand that this radius is larger than $R_{0}$ so that the CSM has both an optically thin and an optically thick part. This requires a slightly different set of fitting parameters: $R_{0}, t_{\mathrm{sh}}, t_{d}, m, \rho_{0}$, and $E_{\mathrm{sh}}$, and we again fix $t_{\mathrm{sh}}=t_{\max }=34$ days. The best fit (plotted in the middle panel of Figure 14) is the same as that obtained in the purely optically thick shell case indicating that any contribution from optically thin emission is negligible. Therefore, for a hybrid model, the optically thick component (Equation (3)) dominates all phases of the observed ROTSE light curve of SN 2008am.

\subsection{A Magnetar-powered SN 2008am?}

Kasen \& Bildsten (2010) and Woosley (2010) proposed the idea that the light curves of some SLSNe may be powered by the spin-down of young magnetars. In such a model, the energy input by the magnetar is given by the dipole spin-down formula:

$$
L_{p}(t)=\frac{E_{p}}{t_{p}} \frac{l-1}{\left(1+t / t_{p}\right)^{l}},
$$

where $E_{p}$ is the initial magnetar rotational energy, $t_{p}$ is the characteristic time scale for spin-down that depends on the strength of the magnetic field and $l=2$ for a magnetic dipole. For a fiducial moment of inertia, the initial period of the magnetar in units of $10 \mathrm{~ms}$ is given by $P_{10}=\left(2 \times 10^{50} \mathrm{erg} \mathrm{s}^{-1} / E_{p}\right)^{0.5}$. The magnetic field of the magnetar can be estimated from $P_{10}$ and $t_{p}$ as $B_{14}=\left(1.3 P_{10}^{2} / t_{p, \mathrm{yr}}\right)^{0.5}$, where $B_{14}$ is the magnetic field in units of $10^{14} \mathrm{G}$ and $t_{p, y r}$ is the characteristic time scale for spin-down in units of years.

Adopting Equation (11) as the energy deposition function (instead of the corresponding one for the radioactive decays of Nickel and Cobalt that leads to the Arnett 1980 solution) and using the first law of thermodynamics coupled with the diffusion approximation, it can be shown that the light curve of a supernova powered by a magnetar is given by the following expression:

$$
L(t)=\frac{E_{p}}{t_{p}} e^{-x^{2} / 2} \int_{0}^{x} e^{z^{2} / 2} \frac{z}{(1+y z)^{2}} d z,
$$

where $x=t / t_{d}$ and $y=t_{d} / t_{p}$ with $t_{d}$ being the characteristic diffusion time. In this treatment, we assume that the initial radius of the progenitor star, $R_{0}$, is small. We fit Equation (12) to the ROTSE light curve of SN 2008am and we obtain $E_{p}=3.2 \times 10^{51} \mathrm{erg}, t_{d}=64$ days and $t_{p}=64$ days. Note that in this model, neither $t_{p}$ nor $t_{d}$ corresponds to the time of maximum light. The fit of this model is shown as the red solid curve in the right panel of Figure 14. Using the fitted values of $E_{p}$ and $t_{p}$, the implied initial period of the magnetar would be $2.5 \mathrm{~ms}$ and the magnetic field $\simeq 0.7 \times 10^{14} \mathrm{G}$. The total radiated energy implied by the magnetar model is $\sim 10^{51} \mathrm{erg}$. The values of $P$ and $B$ lie within the range predicted in Duncan $\&$ Thompson (1992) for magnetars assuming the field to arise in an $\alpha-\Omega$ dynamo. The magnetar model does provide a reasonable fit to the data including the rise time (Figure 14, right panel). Although the magnetar model provides a decent fit to the light curve, it gives no natural explanation for the emission lines.

\section{CONCLUSIONS}

We presented an analysis of the available photometric and spectroscopic data of the SLSN 2008am. The spectroscopic signatures of intermediate-width $\mathrm{H}$ and $\mathrm{He}$ emission lines $(\sim 25 \AA)$ place this SN in the category of Type IIn. SN 2008am was an extremely luminous event, with a peak absolute $R$ magnitude of $M_{R} \simeq-22.3 \mathrm{mag}$ corresponding to a luminosity of $\sim 2 \times 10^{44} \mathrm{erg} \mathrm{s}^{-1}$, putting it in the "hall of fame" of the most luminous SNe ever observed. The host of SN 2008am is a faint extended galaxy with magnitude $\sim 20$ in the SDSS catalog. At the redshift of $z=0.2338$, the absolute $r^{\prime}$ magnitude of the host is $M_{r^{\prime}} \sim-20 \mathrm{mag}$, which is in the range typical for elliptical and spiral galaxies. The very late (+554 day) Keck spectrum of SN 2008am is consistent with an SB1 template spectrum. From the line flux ratios the host has sub-solar metallicity, $Z \sim 0.4 Z_{\odot}$. 
We conclude that the host of SN 2008am is a metal-poor, but normal galaxy, not a subluminous dwarf as is the case for many SLSNe (Miller et al. 2009, 2010; Drake et al. 2010).

SN 2008am was followed up photometrically from the IR to the UV. The ROTSE light curve provides a reasonably accurate estimate of the rise time to maximum light of 34 days in the rest frame, a significantly short time compared to other SLSNe. The photometric coverage allowed us to create broadband SEDs of SN 2008am for six epochs. We fit singletemperature blackbody curves to the SEDs to study the evolution of the blackbody temperature and radius as well as to estimate a pseudo-bolometric light curve. The derived blackbody temperatures $(\sim 10,000-12,000 \mathrm{~K})$ are consistent with the continua of contemporaneous spectra. These temperatures are very high compared with typical core-collapse supernova photospheres $(5000-6000 \mathrm{~K})$ as well as with the temperatures obtained for SN 2006gy and SN 2006tf (6000-8000 K; Smith et al. 2008, 2010). The single-temperature blackbody fits for SN 2008am were imperfect, implying that the underlying emission mechanism is more complex in nature.

Spectra obtained about 10-30 rest-frame days after maximum light showed intermediate-width emission lines of $\mathrm{H} \alpha, \mathrm{H} \beta, \mathrm{H} \gamma$, and a feature at the $\mathrm{He} \mathrm{I} / \mathrm{Na} \mathrm{D}$ blend. There is no sign in our data of broad P Cygni lines that might signify the photosphere of the underlying supernova nor of narrow $\mathrm{P}$ Cygni lines as displayed by SN 2006gy and SN 2008tf on decline that indicate absorption in the unshocked, but expanding circumstellar matter (Smith et al. 2008, 2010). A spectrum obtained 352 days after our estimated maximum showed only $\mathrm{H} \alpha$ that was significantly narrower than in the earlier spectra. Our failure to detect P Cygni features is most likely attributable to our lack of data at phases fainter than 2 mag from maximum when SN 2006gy began to show such features, although we cannot rule out issues of $\mathrm{S} / \mathrm{N}$ and wavelength resolution. The overall observed spectral evolution of SN 2008am is similar to that of SN 2006gy at similar phases, and we conclude that they are closely related.

We considered a variety of models for the emission line profiles: Gaussian as might typify thermal Doppler broadening, an exponential profile that might characterize single electron scattering in an optically thin medium, and Lorentzian that might represent models of multiple electron scattering (Chugai 2001; Smith et al. 2010). We find that the latter provides the best fit to the overall line shape. An important implication is that the line broadening is probably dominated by electron scattering in our spectra and that the line width contains little or no information about the bulk kinetic expansion velocity of the matter in the CSM or the underlying supernova. An upper limit to the velocity in the line-forming region is about $1000 \mathrm{~km} \mathrm{~s}^{-1}$. Chugai made specific assumptions in his models, for instance, that the velocity profile decreased outward as might result from radiative acceleration, that might not apply in general. The line profiles might contain information about the velocity structure even if scattering dominated (Fransson \& Chevalier 1989). The radiative transfer that results in these line profiles is worthy of re-examination.

We explored a number of light curve models based on a generalization of the models of Arnett $(1980,1982)$ that use a specified power input and the first law of thermodynamics coupled with the diffusion equation. The models are constrained by the rise time, the quasi-bolometric light curve, and the general nature of the emission line spectra that show intermediate-width lines with little or no sign of P Cygni absorption on any scale. We examined models based on radioactive decay, an underlying supernova striking an optically thick circumstellar shell, a supernova shock in an optically thin CSM, and a magnetar. The radioactive decay model is ruled out because the required nickel mass would exceed the deduced ejecta mass. The magnetar model provides a decent fit to the light curve, but no natural explanation for the emission-line structure. A shell-shock model similar to that of Smith \& McCray (2007) for SN 2006gy in which the diffusion times on the rise and the decline are equal fails drastically for SN 2008am, primarily because of the rapid rise, 34 days in the rest frame. We conclude that the success of a model with a single diffusion time for SN 2006gy was a coincidence and not a general property of this class of events. We could generate a reasonable fit in the context of this model by including an input power source representing the collision of the supernova with an optically thick shell that was a "top hat" function of constant luminosity during the rise that shut off at 34 days, the point of maximum light in this model. In this model, the timescale of the power source dictated the rise time and a separately determined diffusion time of 120 days governed the decline. This model rose with $L \propto t^{2}$ at very early times (as assumed for the full rise of SN 2006gy by Smith \& McCray) and was overall concave upward in contrast to the observed light curve that appears to be concave downward. The shape of the rise depends on the input power profile. We will investigate more general models in a future paper. The optically thin model is not an Arnett-like diffusion model, but assumes that the shock energy is rapidly radiated. This model did not provide a selfconsistent fit to SN 2008am and gives no natural explanation for the rise nor for the failure to see any high-velocity features corresponding to the photosphere of the underlying supernova.

The best fit to the ROTSE light curve data was obtained with an ejecta-CSM interaction model in which the supernova is not seen directly and the luminosity is produced by shocks and diffusion in a CSM that is optically thick. For fiducial parameters of $v=1000 \mathrm{~km} \mathrm{~s}^{-1}$ and $\kappa=0.4 \mathrm{~cm}^{2} \mathrm{~g}^{-1}$, this model gives a rather small initial radius for the shell, $R_{0} \sim 1.0 \times 10^{14} \mathrm{~cm}$, with a mass of about $1 M_{\odot}$ and a total energy input from the underlying SN/CSM shock of $E_{s}=5.5 \times 10^{51} \mathrm{erg}$. The model suggests that the optically thick component may dominate the luminosity and that the forward shock provides a greater contribution to the luminosity than the reverse shock. This model suggests a rather large mass-loss rate for the progenitor, as perhaps would be consistent with an LBV-type mass-loss process.

As noted above, SN 2008am seems to be a close cousin of SN 2006gy, showing Lorentzian emission lines shortly after maximum and a narrower $\mathrm{H} \alpha$ line about a year later. The Lorentzian lines in both events show a slight redshift of about $100 \mathrm{~km} \mathrm{~s}^{-1}$. The most notable difference is our failure to see the distinct broader $\left(4000 \mathrm{~km} \mathrm{~s}^{-1}\right)$ and narrow $\left(200 \mathrm{~km} \mathrm{~s}^{-1}\right)$ $\mathrm{P}$ Cygni features that appeared in SN 2006gy 20 to 80 days after maximum light. Smith et al. (2010) attribute the first phase of pure emission to conditions where the shock is still beneath the photosphere of the dense CSM and the second phase where P Cygni features form to conditions where the shock has proceeded beyond the photosphere. The high-velocity absorption is presumably related to the SN ejecta, and the lowvelocity absorption to the motion of the CSM that has not yet been hit by the shock, but is subject to radiative excitation and recombination. The most likely explanation of our failure to detect these P Cygni features is absence of data at the appropriate phase. The narrower $\mathrm{H} \alpha$ line in both events nearly a year after explosion is consistent with the emitting matter 
becoming more dilute with less broadening by multiple electron scattering.

Other SLSNe seem to fall broadly in the category of SN 2006gy and SN 2008am. SN 2006tf shows nearly symmetric emission lines, especially of $\mathrm{H} \alpha$, up to 40 days after discovery (there is no data on the rise so the explosion date and date of maximum are uncertain), with the development of narrow $\mathrm{P}$ Cygni features by 66 days after discovery (Smith et al. 2008). At these later epochs, there are indications in both the emission and absorption for rapidly moving material, $\sim 7500 \mathrm{~km} \mathrm{~s}^{-1}$, presumably from the underlying SN ejecta. SN 2006tf is somewhat different from SN 2006gy and SN 2008am in the late phases, a year after explosion, where the $\mathrm{H} \alpha$ line seems to be formed by collisional rather than radiative excitation (Smith et al. 2008). The $\mathrm{H} \alpha$ line in SN 2006tf at this stage shows a prominent blue "plateau" extending to about $1000 \mathrm{~km} \mathrm{~s}^{-1}$. SN 2008iy was an unusual SN IIn with the unprecedented slow rise time of 400 days (Miller et al. 2010). The post-maximum spectra of SN 2008iy are somewhat similar to those of SN 2008am, with strong intermediate-width $\mathrm{H}$ and $\mathrm{He}$ emission lines. A singletemperature blackbody failed to provide a good fit to the SEDs of SN 2008iy, as we found for the SED of SN 2008am (Section 2.3). Miller et al. identified three distinct components in the late-time $\mathrm{H} \alpha$ profile of SN 2008iy: broad $\left(\sim 4500 \mathrm{~km} \mathrm{~s}^{-1}\right)$, intermediate $\left(\sim 1650 \mathrm{~km} \mathrm{~s}^{-1}\right)$, and narrow $\left(\sim 75 \mathrm{~km} \mathrm{~s}^{-1}\right)$. Miller et al. proposed a model of interaction of the SN ejecta with a clumpy CSM similar to that for SN 1988 Z presented by Chugai \& Danziger (1994). Miller et al. argued that the rise in the light curve resulted from an increase in the number of clumps with radius. Given the success of the shell-shock models of SN 2006gy and SN 2008am, it would be of interest to apply such a model to SN 2008iy.

As for SN 2006gy and SN 2006tf, the suggestion of a relatively massive shell around SN 2008am and the estimated mass-loss rates $\left(\sim 0.1-10 M_{\odot} \mathrm{yr}^{-1}\right)$ imply that the progenitor star must have undergone substantial mass loss in the years prior to the explosion. Episodic mass loss can occur around very massive LBV stars, similar to $\eta$ Carinae. The reason for LBV mass loss is not currently fully understood (Smith \& Owocki 2006). Massive shell ejection can also be the product of pulsational pair-instability (Rakavy \& Shaviv 1967; Barkat et al. 1967). Models suggest that repetitive shell ejection takes place for progenitor main sequence masses in the range 95-130 $M_{\odot}$ (Woosley et al. 2007). Supernova-like luminosity can be produced either during the ejection of each of these shells individually (Kasen et al. 2008), or during the collisions between shells ejected at different times (Woosley et al. 2007).

Although there is some sign of high-velocity material in some of the SLSNe that otherwise resemble SN 2008am, the nature of the presumed underlying supernova in these Type IIn SLSNe remains obscure. As noted in the introduction, other SLSNe show little or no evidence for hydrogen or interaction with a CSM. In the case of SN 2007bi, this is an important part of the argument that it is a pair-instability supernova (Gal-Yam et al. 2009). An important goal in the study of SLSNe remains the determination of the density distribution in the CSM that will give important clues to the mass-loss history.

We are grateful to the anonymous referee for valuable guidance on style and science, and to Andy Howell and Milos Milosavljevic for useful discussions. This research is supported in part by NSF Grant AST-0707669 and by the Texas Advanced Research Program grant ASTRO-ARP-0094. E.C. thanks the
Propondis foundation of Piraeus, Greece for its support of his studies. J.V. received support from Hungarian OTKA Grant K76816.

\section{REFERENCES}

Akerlof, C. W., et al. 2003, PASP, 115, 132

Aldering, G., et al. 2006, ApJ, 650, 510

Aretxaga, I., Benetti, S., Terlevich, R. J., Fabian, A. C., Cappellaro, E., Turatto, M., \& della Valle, M. 1999, MNRAS, 309, 343

Arnett, W. D. 1980, ApJ, 237, 541

Arnett, W. D. 1982, ApJ, 253, 785

Arnett, W. D., \& Fu, A. 1989, ApJ, 340, 396

Barbary, K., et al. 2009, ApJ, 690, 1358

Barkat, Z., Rakavy, G., \& Sack, N. 1967, Phys. Rev. Lett., 18, 379

Bloom, J. S., Starr, D. L., Blake, C. H., Skrutskie, M. F., \& Falco, E. E. 2006, in ASP Conf. Ser. 351, Astronomical Data Analysis Software and Systems XV, ed. C. Gabriel et al. (San Francisco, CA: ASP), 751

Botticella, M. T., et al. 2010, ApJ, 717, L52

Burrows, D. N., et al. 2005, Space Sci. Rev., 120, 165

Cenko, S. B., et al. 2006, PASP, 118, 1396

Cenko, S. B., et al. 2009, ApJ, 693, 1484

Chandra, P., \& Soderberg, A. 2008, ATel, 1408, 1

Chatzopoulos, E., Wheeler, J. C., \& Vinko, J. 2009, ApJ, 704, 1251

Chevalier, R. A. 1982, ApJ, 258, 790

Chevalier, R. A., \& Fransson, C. 1994, ApJ, 420, 268

Chevalier, R. A., \& Fransson, C. 2003, in Supernovae and Gamma-ray Bursters, ed. K. Weiler (Lecture Notes in Physics, vol. 598; Berlin: Springer), 171 Chugai, N. N. 2001, MNRAS, 326, 1448

Chugai, N. N., \& Danziger, I. J. 1994, MNRAS, 268, 173

Chugai, N. N., et al. 2004, MNRAS, 352, 1213

Clocchiatti, A., et al. 2000, ApJ, 529, 661

Dessart, L., Hillier, D. J., Gezari, S., Basa, S., \& Matheson, T. 2009, MNRAS, 394,21

Drake, A. J., et al. 2010, ApJ, 718, L127

Duncan, R. C., \& Thompson, C. 1992, ApJ, 392, L9

Filippenko, A. V. 1989, AJ, 97, 726

Fitzpatrick, E. L., \& Massa, D. 2007, ApJ, 663, 320

Fox, O. D., Chevalier, R. A., Dwek, E., Skrutskie, M. F., Sugerman, B. E. K., \& Leisenring, J. M. 2010, ApJ, 725, 1768

Fransson, C. 1984, A\&A, 132, 115

Fransson, C., \& Chevalier, R. A. 1989, ApJ, 343, 323

Fukugita, M., Ichikawa, T., Gunn, J. E., Doi, M., Shimasaku, K., \& Schneider, D. P. 1996, AJ, 111, 1748

Gal-Yam, A., et al. 2009, Nature, 462, 624

Gezari, S., et al. 2009, ApJ, 690, 1313

Hamuy, M., et al. 2003, Nature, 424, 651

Hatano, K., Branch, D., Nomoto, K., Deng, J. S., Maeda, K., Nugent, P., \& Aldering, G. 2001, BAAS, 33, 838

Hill, G. J., Nicklas, H. E., MacQueen, P. J., Tejada, C., Cobos Duenas, F. J., \& Mitsch, W. 1998, Proc. SPIE, 3355, 375

Immler, S., \& Kuntz, K. D. 2005, ApJ, 632, L99

Kasen, D., \& Bildsten, L. 2010, ApJ, 717, 245

Kasen, D., Heger, A., \& Woosley, S. 2008, in AIP Conf. Proc. 990, First Stars III, ed. B.W. O'Shea, A. Heger, \& T. Abel (Melville, NY: AIP), 263

Kinney, A. L., Calzetti, D., Bohlin, R. C., McQuade, K., Storchi-Bergmann, T., \& Schmitt, H. R. 1996, ApJ, 467, 38

Kotak, R., et al. 2009, ApJ, 704, 306

Laor, A. 2006, ApJ, 643, 112

Mattila, S., et al. 2008, MNRAS, 389, 141

Meikle, W. P. S., et al. 2007, ApJ, 665, 608

Miller, A. A., et al. 2009, ApJ, 690, 1303

Miller, A. A., et al. 2010, MNRAS, 404, 305

Moriya, T., Tominaga, N., Tanaka, M., Maeda, K., \& Nomoto, K. 2010, ApJ, 717, L83

Neill, J. D., et al. 2011, ApJ, 727, 15

Ofek, E. O., et al. 2007, ApJ, 659, L13

Oke, J. B., et al. 1995, PASP, 107, 375

Osterbrock, D. E. 1989, Astrophysics of Gaseous Nebulae and Active Galactic Nuclei (Mill Valley, CA: Univ. Science Books)

Pastorello, A., et al. 2002, MNRAS, 333, 27

Pastorello, A., et al. 2008, MNRAS, 389, 131

Pastorello, A., et al. 2010, ApJ, 724, L16

Pettini, M., \& Pagel, B. E. J. 2004, MNRAS, 348, L59

Poole, T. S., et al. 2008, MNRAS, 383, 627

Quimby, R. 2006, CBET, 644, 1 
Quimby, R. M., Aldering, G., Wheeler, J. C., Höflich, P., Akerlof, C. W., \& Rykoff, E. S. 2007a, ApJ, 668, L99

Quimby, R. M., Castro, F., Gerardy, C. L., Hoeflich, P., Kannappan, S. J., Mondol, P., Sellers, M., \& Wheeler, J. C. 2005, BAAS, 37, 1431

Quimby, R., Castro, F., \& Mondol, P. 2007b, IAU Circ., 8790, 2

Quimby, R., Castro, F., Mondol, P., Caldwell, J., \& Terrazas, E. 2007c, CBET, 793, 1

Quimby, R., Yuan, F., Chatzopoulos, M., Vinko, J., Akerlof, C., \& Wheeler, J. C. 2009, American Astronomical Society Meeting Abstracts, 214, 427.01

Rakavy, G., \& Shaviv, G. 1967, ApJ, 148, 803

Rest, A., et al. 2009, arXiv:0911.2002

Roming, P. W. A., et al. 2005, Space Sci. Rev., 120, 95

Schlegel, E. M. 1990, MNRAS, 244, 269

Schlegel, D. J., Finkbeiner, D. P., \& Davis, M. 1998, ApJ, 500, 525

Skrutskie, M. F., et al. 2006, AJ, 131, 1163

Smith, N., Chornock, R., Li, W., Ganeshalingam, M., Silverman, J. M., Foley, R. J., Filippenko, A. V., \& Barth, A. J. 2008, ApJ, 686, 467

Smith, N., Chornock, R., Silverman, J. M., Filippenko, A. V., \& Foley, R. J. 2010, ApJ, 709, 856

Smith, N., \& McCray, R. 2007, ApJ, 671, L17
Smith, N., \& Owocki, S. P. 2006, ApJ, 645, L45

Smith, D., et al. 2003, in AIP Conf. Proc. 662, Gamma-Ray Burst and Afterglow Astronomy 2001: A Workshop Celebrating the First Year of the HETE Mission, ed. G. R. Ricker \& R. K. Vanderspek (Melville, NY: AIP), 514

Smith, N., et al. 2007, ApJ, 666, 1116

Soderberg, A. M., et al. 2008, Nature, 453, 469

Stathakis, R. A., \& Sadler, E. M. 1991, MNRAS, 250, 786

Stoll, R., et al. 2010, arXiv:1012.3461

Thuan, T. X., \& Gunn, J. E. 1976, PASP, 88, 543

Turatto, M., Cappellaro, E., Danziger, I. J., Benetti, S., Gouiffes, C., \& della Valle, M. 1993, MNRAS, 262, 128

Umeda, H., \& Nomoto, K. 2008, ApJ, 673, 1014

Valenti, S., et al. 2008, MNRAS, 383, 1485

Woosley, S. E. 2010, ApJ, 719, L204

Woosley, S. E., Blinnikov, S., \& Heger, A. 2007, Nature, 450, 390

Young, T. R., Smith, D., \& Johnson, T. A. 2005, ApJ, 625, L87

Young, D. R., et al. 2010, A\&A, 512, A70

Yuan, F., et al. 2007, BAAS, 38, 929

Yuan, F., et al. 2008a, CBET, 1262, 1

Yuan, F., et al. 2008b, CBET, 1462, 1 